\title{
PHYTOSOCIOLOGICAL DESCRIPTION OF QUERCUS PETRAEA FOREST STANDS WITH CHAMAECYTISUS HIRSUTUS AND ERICA CARNEA IN THE VIPAVSKA BRDA (SOUTHWESTERN SLOVENIA) FITOCENOLOŠKA OZNAKA SESTOJEV GRADNA (QUERCUS PETRAEA) Z DLAKAVO RELIKO (CHAMAECYTISUS HIRSUTUS) IN SPOMLADANSKO RESO (ERICA CARNEA) V VIPAVSKIH BRDIH (JUGOZAHODNA SLOVENIJA)
}

\author{
Igor DAKSKOBLER ${ }^{1}$ \\ (1) Institute of Biology, Scientific Research Centre of the Slovenian Academy of Sciences and Arts, Regional Unit Tolmin, Brunov \\ drevored 13, SI-5220 Tolmin, and Biotechnical Faculty of the University in Ljubljana, Department of Forestry and Renewable \\ Forest Resources, Večna pot 83, 1000 Ljubljana, igor.dakskobler@zrc-sazu.si
}

\begin{abstract}
We conducted a phytosociological study of Quercus petraea stands, whose herb layer is dominated by Erica carnea in the flysch hills of Vipavska brda and on the margins of the Vrhe plateau (southwestern Slovenia). We have determined that they are a long-term degradation stage on beech forest sites from the association Seslerio autumnalis-Fagetum. Based on comparisons with similar sessile oak stands from associations Melampyro vulgati-Quercetum petraeae, Seslerio autumnalis-Quercetum petraeae and Erico carneaeQuercetum petraeae, the studied stands are classified into the new association Chamaecytiso hirsuti-Quercetum petraeae. They are best differentiated from the stands of compared communities by the species Erica carnea, Sorbus aria, Lathyrus linifolius, Loranthus europaeus and Erythronium dens-canis. The new association is classified into the alliance Carpinion orientalis and order Quercetalia pubescenti-petraeae.
\end{abstract}

Key words: phytosociology, synsystematics, Seslerio autumnalis-Fagetum, Chamaecytiso hirsuti-Quercetum petraeae, Carpinion orientalis, degradation stage, Vipavska brda, Vrhe, Slovenia

\begin{abstract}
IZVLEČEK
Fitocenološko smo raziskali sestoje gradna (Quercus petraea) v flišnem gričevju Vipavskih brd in na robu planote Vrhe (jugozahodna Slovenija), v katerih v zeliščni plasti prevladuje vrsta Erica carnea. Ugotavljamo, da so dolgotrajen degradacijski stadij na rastiščih bukovega gozda iz asociacije Seslerio autumnalis-Fagetum. Na podlagi primerjav s podobnimi gradnovimi sestoji iz asociacij Melampyro vulgati-Quercetum petraeae, Seslerio autumnalis-Quercetum petraeae in Erico carneae-Quercetum petraeae preučene sestoje uvrščamo v novo asociacijo Chamaecytiso hirsuti-Quercetum petraeae. Od sestojev primerjanih združb jih najbolj razlikujejo vrste Erica carnea, Sorbus aria, Lathyrus linifolius, Loranthus europaeus in Erythronium dens-canis. Novo asociacijo uvrščamo v zvezo Carpinion orientalis in v red Quercetalia pubescenti-petraeae.
\end{abstract}

Ključne besede: fitocenologija, sinsistematika, Seslerio autumnalis-Fagetum, Chamaecytiso hirsuti-Quercetum petraeae, Carpinion orientalis, degradacijski stadij, Vipavska brda, Vrhe, Slovenija

GDK 188:176.1Quercus petraea(497.4)(045)=111 Prispelo / Received: 22. 02. 2014

Sprejeto / Accepted: 24.04. 2014

\section{INTRODUCTION}

1 UVOD

The forest of the Vipavska brda in southwestern Slovenia as seen from a distance (e.g. from the Nanos plateau) gives a fairly uniform picture. A closer view, however, shows that these are secondary stands that clearly demonstrate the impact of past management. In terms of ecology - these are steep, shady flysch slopes cross-cut with streams - the highest stage of development in the primary post-glacial development in this area was probably beech forest. Individual beech trees can still be found in numerous spots, as well as many beech stands, especially on higher elevations on slopes under the Vrhe plateau and in the eastern part of the hills towards Razdrto and Raša. We conducted a phytosociological inventory of several such beech stands that are mainly classified into the association Seslerio autumnalis-Fagetum (compare Dakskobler, 1997). The dominant species now is without a doubt sessile oak (Quercus petraea), and its stands, whose herb layer is dominated by autumn moor grass ( $\mathrm{Se}$ sleria autumnalis), are classified into the association Seslerio autumnalis-Quercetum petraeae (Čarni et al., 2002). We were especially interested in low-growing oak coppice stands, where the herb layer is completely dominated by spring heath (Erica carnea). Elevation, geological bedrock, slope are similar as in beech or sessile oak stands with autumn moor grass, while the 
herb layer is clearly different. Degradation and, in turn, deterioration of soil- and consequently of site conditions and changes in the species composition could have been caused by erosion (removal of soil still ongoing in several locations) and definitely also by past human activities, especially by litter gathering (Čokelj, in litt.). A phytosociological inventory was conducted for both beech and oak stands; the relevés were arranged into two tables and the results of our research are presented in this paper.

\subsection{Ecological description of the study area}

\subsection{Ekološki opis raziskovanega območja}

The name Vipavska brda denominates the hills situated south of the Vipava river, north of the Branica river and west of the Močilnik stream (Kladnik, 2000) in southwestern Slovenia. In the east, the hills gradually continue into the Vrhe plateau between the Vipava and Raša valleys. The shady slopes of the Vipavska brda and of the Vrhe plateau above the Močilnik and Pasji rep streams are now mainly covered with forests. Settlements are located both on the plateau and in the valley. Figure 1 shows the approximate location of inventoried sessile oak stands, whose herb layer is completely dominated by spring heath (Erica carnea). The elevation of the localities is between $290 \mathrm{~m}$ and $535 \mathrm{~m}$, the aspect is distinctly shady, northern, northeastern, eastern as well as southeastern, the slope is $5^{\circ}$ to $30^{\circ}$. Geological bedrock of the Vipavska brda is Eocene flysch (Buser, 1973, 2009) and the predominant soil type is eutric brown soil (Lovrenčak, 1998, Prus, in litt.).

The climate is warm, with mean annual temperature of $10^{\circ} \mathrm{C}$ to $12^{\circ} \mathrm{C}$ (Cegnar, 1998) and mean annual precipitation of between 1,400 $\mathrm{mm}$ and 1,600 $\mathrm{mm}$ (B. Zupančič, 1998). The phytosociological map of potential natural forest vegetation of Slovenia, scale 1: 400,000 (Čarni et al., 2002), indicates the study area as the association Seslerio autumnalis-Quercetum petrae$a e$. According to our findings, beech forest is the potential natural vegetation of shady slopes of the Vipavska brda, especially the forest from the association Seslerio autumnalis-Fagetum, in part also from the association Ornithogalo pyrenaici-Fagetum. Stands of the association Seslerio autumnlais-Quercetum petraeae (see relevés 11 and 12 in Table 2) in this area are largely secondary degradation stage. Although the steep flysch slopes have always been largely wooded, the military map from the second half of the 18th century (Rajšp and Trpin, 1997) indicates relatively large viticultural areas also on the shady slopes, at least in the Pasji rep

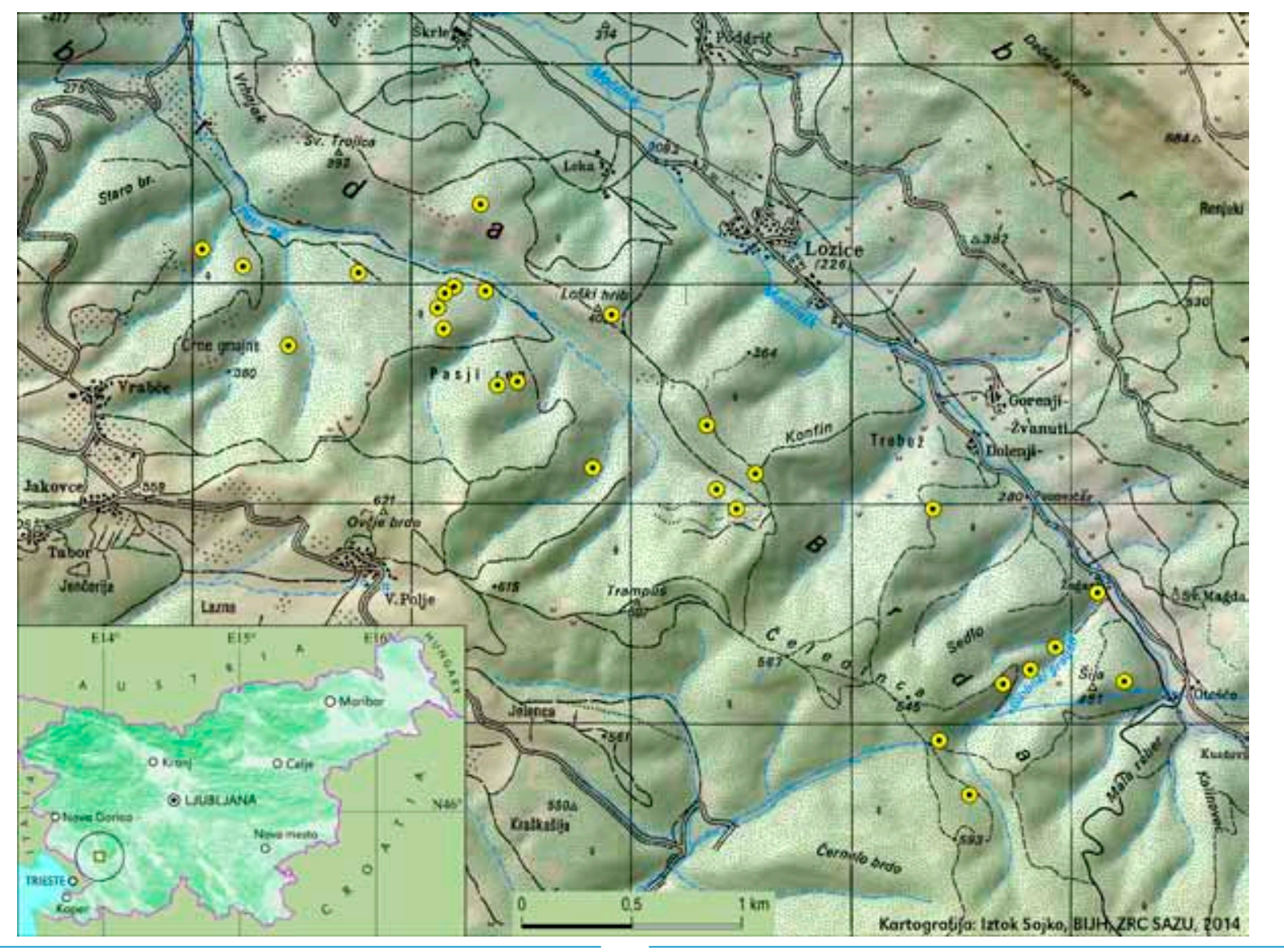

Fig. 1: Approximate localities of inventoried sessile oak stands in the Vipavska brda

Slika 1: Približna nahajališča raziskanih gradnovih sestojev v Vipavskih brdih 
valley. Pioneer stands of various deciduous trees occur elsewhere too, especially in gullied slopes with deep soil. They frequently feature wild cherry (Prunus avium) - see relevé No. 13 in Table 2. Sites on what was once clearly agricultural land are increasingly overgrown by black locust (Robinia pseudoacacia).

\section{MATERIALS AND METHODS}

\section{MATERIALI IN METODE}

Vegetation in the Vipavska brda was researched applying the Central-European phytosociological method (Braun-Blanquet, 1964). A total of 39 relevés were made and entered into the FloVegSi database (Seliškar et al., 2003). Combined cover-abundance values were transformed into numerical values 1- 9 (van der Maarel, 1979). Numerical comparisons were conducted with the software package SYN-TAX (Podani, 2001). Relevés were arranged into two analytic tables (Tables 1 and 2) based on hierarchical classification. We integrated the results of the (unweighted) pair group method with arithmetic mean "(Unweighted) average linkage" - UPGMA, where Wishart's similarity ratio was applied. Phytosociological groups (= groups of diagnostic species) were formed on the basis of our own criteria, but with consideration of several authors (Aeschimann et al., 2004, Poldini, 1982, 1988, 1989, Zupančič, 1999). Similar oak communities from the sub-Mediterranean part of Slovenia (Poldini, 1982, Zupančič, 1999) and from serpentine areas in Bosnia (Krause and Ludwig, 1957, Ritter-Studnička,1970) were compared through hierarchical classification and two-dimensional ordination (principal coordinates analysis, PCoA), as well as on the basis of Sørensen's coefficient of floristic similarity (Sørensen, 1948) and analysis of percentage of diagnostic species of syntaxonomic groups. The nomenclature source for the names of vascular plants is Mala flora Slovenije (Martinčič et al., 2007). Martinčič $(2003,2011)$ is the nomenclature source for the names of mosses, Šilc and Čarni (2012) for the names of syntaxa (with the exception of the names of the class Querco-Fagetea Braun-Blanquet et Vlieger in Vlieger 1937 and alliance Carpinion orientalis Horvat 1958) and Urbančič et al. (2005) for the names of soil types.

\section{RESULTS AND DISCUSSION 3 REZULTATI IN RAZPRAVA}

\subsection{Description of soils in the researched stands}

3.1 Opis tal v preučenih sestojih

Soil conditions were described on the basis of the sample that was obtained in the Pasji rep valley and analysed in the laboratory of the Slovenian Forestry Institute, as well as on the basis of the public data provided by the Infrastructural Centre for Pedology and Plant Protection at the Department of Agronomy of Biotechnical Faculty in Ljubljana, which were interpreted for us by Tomaž Prus (in litt.). Convex slopes with relatively shallow soil prevail, in places showing signs of erosion (leaching). The dominant soil type is lessivé eutric brown soil. Upper horizons are strongly acid ( $\mathrm{pH} \mathrm{H}_{2} \mathrm{O} 4.8, \mathrm{pH} \mathrm{CaCl} 3.8$ to 4.0), the humus type is moder (C/N ratio exceeds 20$)$. Soil properties change with depth and $\mathrm{pH}$ increases considerably. This indicates past practices on surface soil horizons. Litter gathering is one of the likely reasons that accelerated the process of soil base cation ( $\mathrm{Ca}, \mathrm{Mg}, \mathrm{K}$ ) leaching. $\mathrm{Al}$ though characteristic for alkaline soils, Erica carnea can thrive also on soils that have gradually become acid. In the event of further soil degradation (acidification), it would be replaced by Calluna vulgaris, which has only been found in isolated spots in our relevés. Abundant occurrence of Erica carnea in the Vipavska brda can be attributed to the distinctly shady aspect, i.e. local climate factors, erosion-associated processes on convex slopes and to past litter gathering.

\subsection{Stand description and species composition (Table 1)}

3.2 Oznaka sestojev in vrstna sestava (preglednica 1)

Pole- and mature sessile oak stands prevail on 39 researched plots; their diameter at breast height is between $25 \mathrm{~cm}$ and $35 \mathrm{~cm}$ and tree height between $10 \mathrm{~m}$ and $16 \mathrm{~m}$. Many of them are of coppice origin. Frequent occurrence of the parasite Loranthus europaeus in sessile oak crowns indicates relatively poor vitality of sessile oak on these sites. The upper tree layer is admixed with individual trees of Fagus sylvatica, Castanea sativa and Sorbus aria, very rarely also by Pinus nigra (subspontaneously), Betula pendula, Ostrya carpinifolia, Sorbus aucuparia and Pinus sylvestris. The alien species Robinia pseudoacacia was recorded in the tree layer on one of the plots. The species that frequently occur in the lower tree layer and in the upper shrub layer include Fraxinus ornus, Sorbus aria, S. torminalis, Juniperus communis, in places also Castanea sativa and Fagus sylvatica, and only rarely Laburnum alpinum, Pyrus pyraster, Amelanchier ovalis and Crategus monogyna. In the shrub layer, we inventoried the taxa Sorbus graeca and $S$. austriaca, but our determination is not completely reliable. Above Veliki graben above Otošče we found a rare hybrid between Sobus aria and S. torminalis $=$ Sorbus latifolia s. lat. In addition to the dominating Erica 
carnea, the taxon Molinia caerulea subsp. arundinacea, in places also the species Convallaria majalis, Serratula tinctoria and Vaccinium myrtillus, on several spots also Pteridium aquilinum, have a higher medium coverage. Common grasses include Sesleria autumnalis, Avenella flexuosa and Calamagrostis arundinacea; Luzula luzuloides is also frequent. Species with high constancy (more than 50\%) in the herb layer are also Chamaecytisus hirsutus, Tanacetum corymbosum, Hieracium racemosum, $H$. sabaudum, $H$. murorum, Genista pilosa, Platanthera bifolia and Erythronium dens-canis. Common species in the moss layer are Hypnum cupressiforme, Thuidium tamariscinum and Polytrichum formosum (= Polytrichastrum formosum), with Leucobryum glaucum observed on several plots.

\subsection{Comparison with preserved beech stands} (Table 2)

3.3 Primerjava z ohranjenimi bukovimi sestoji (preglednica 2)

Table 2 comprises 13 relevés from the same area (Vipavska brda, Vrhe), but with a different composition of the tree layer. Ten relevés (1 to 10) are classified into the association Seslerio autumnalis-Fagetum, two are a degradation stage with dominant sessile oak on a beech site (relevés 11 and 12, both classified into the association Seslerio autumnalis-Quercetum petraeae). Relevé No. 13 characterises a pioneer forest on former agricultural land dominated by Prunus avium, with individual specimens of Ostrya carpinifolia, Acer pseudoplatanus, A. campestre and Robinia pseudoacacia. We assume this is a pioneer stage on potential sites of the association Ornithogalo pyrenaici-Fagetum. Comparison of 26 relevés of sessile oak forest with ten relevés of the beech forest from the association Seslerio autumnalis-Fagetum from the same area indicates substantial differences not only in the tree layer, but also in the herb layer. In beech stands, it is usually dominated by Sesleria autumnalis, there are clearly more mesophilous species, character species of the order Fagetalia sylvaticae (see Tables 2 and 4). The soil is deeper, less acid and in places also moister.

\subsection{Synsystematic classification}

\subsection{Sinsistematska opredelitev}

Natural characteristics (flysch slopes), phytogeographical position and origin (secondary community) of the studied stands are similar to the conditions in which sessile oak stands of the syntaxon Melampyro vulgati-Quercetum petraeae var. geogr. Fraxinus ornus (Puncer and Zupančič, 1979, Zupančič, 1994) grow in the hills of Brkini. We conducted a comparison between
19 relevés of this association and our relevés; floristic similarity according to Sørensen (1948) is about 56\%, which allows for classification into the same association, but new subassociation Melampyro-Quercetum ericetosum carneae. Such classification is conditionally supported also by the presence of diagnostic species from the association Melampyro-Quercetum in the studied stands in the Vipavska brda. In addition to sessile oak, Luzula luzuloides, Hieracium sabaudum and the geographical differential species Avenella flexuosa (= Deschampsia flexuosa) also frequently occur in these stands. Melampyrum pratense subsp. vulgatum and Chamaecytisus supinus occur with considerably lower frequency and medium coverage, whereas Lembotropis nigricans and geographical differential species Orobanche nana (O. ramosa subsp. nana) were not recorded. There are obvious differences in the proportion of diagnostic species (Table 4). The studied stands comprise a considerably higher proportion of thermophilous species of the order Quercetalia pubescentipetraeae and class Erico-Pinetea and a much smaller proportion of acidophilous species of the order Quercetalia roboris and class Vaccinio-Piceetea than the stands of the association Melampyro-Quercetum. Soil conditions are different. Dystric brown soils prevail in the stands of the association Melampyro-Quercetum, whereas the soil in our stands is eutric, although frequently leached. The fundamental difference is in potentially natural vegetation. In terms of the association Melampyro-Quercetum, this is most frequently a moderate acidophilous beech-oak forest from the association Castaneo-Fagetum sylvaticae, in our case the sub-Mediterranean beech forest from the association Seslerio autumnalis-Fagetum. This is also the basis for classification into two different orders. The association Melampyro-Quercetum is classified into the alliance Quercion roboris (or Genisto germanicae-Quercion roboris) and into the order Quercetalia roboris, while the studied stands with their floristic composition are classified into the alliance Carpinion orientalis and into the order Quercetalia pubescenti-petraeae. If these stands were classified as the new subassociation MelampyroQuercetum petraeae ericetosum carneae it should be classified into the same alliance as the previously described subassociations (Puncer and Zupančič, 1979), i.e. into the alliance Quercion roboris (or Genisto germanicae-Quercion roboris), which does not correspond to its full floristic composition. We therefore conducted additional comparisons with another two syntaxa, the association Seslerio autumnalis-Quercetum petraeae (Zupančič, 1999, Table 5, Poldini, 1982, Table 4, columns 27-34) and with the association Erico carne- 
ae-Quercetum petraeae (Krause and Ludwig, 1957: 118-120, Ritter-Studnička, 1970: 114-115, Beus, 1997: 94-95). We also made a synthetic table (Table 3) with six columns and compared them with hierarchical classification and two-dimensional ordination (Figures 2 and 3). In terms of floristics, our stands are most similar to the stands of the association Melampyro-Quercetum petraeae. Floristic similarity with the association Seslerio autumnalis-Queretum petraeae according to Sørensen (1948) is already lower at about $45 \%$. Similarity is only slightly higher (47\%) with the most acidophilous form of this association described by Poldini (1982) as Seslerio autumnalis-Queretum petraeae avenelletosum flexuosae. It is differentiated by Castanea sativa, Luzula luzuloides, Avenella flexuosa and some other species. Poldini (ibid.) described two variants: the variant with Erica carnea on sites with less acid soil and the variant with Polytrichum formosum, Leucobryum glaucum, Genista germanica and Dicranum scoparium on more acid soil. He found such stands both on flysch and limestone. The tree layer is frequently dominated by Turkey oak (Quercus cerris). Poldini attributed the increased soil acidity to the past litter gathering. Despite certain similarities with the studied stands in the Vipavska brda (occurrence of Erica carnea, but with less medium coverage, and of some other acidophilous species except Vaccinium myrtillus), the dendrogram (Figures 2 and 3 ) shows the stands of the subassociation -avenelletosum flexuosae grouping with stands of other forms of the association Seslerio autumnalis-Quercetum petraeae and not with our community.

With consideration of the dominant species of the tree and herb layers, the studied stands could be classified into the association Erico carneae-Quercetum petraeae that was described in northern Bosnia (Krause and Ludwig, 1957: 90-91 and Table 1, column 3 on pages 118-120, Horvat, 1959: 30, Ritter-Studnička, 1970), but in very different ecological conditions, on serpentine bedrock (in western Bosnia the oak stands with Erica carnea were described also on Permian sandstones and on dystric soils and were classified into the syntaxon Betulo-Quercetum petraeae ericetosum Fabijanić et al., 1963, Stefanović, 1984). Stands of this association are syndynamically closely related to the black and red pine on serpentinite (see also Horvat et al., 1974: 461) and their progressive development also leads to the (fir)beech forest. Floristic similarity of 23 relevés from the synthetic table of the association Erico-Quercetum petraeae Krause et Ludwig ex Horvat 1959 (the correct author citation, Theurillat, in litt.) Ritter-Studnička (1970: 114-115) with our stands is only $20 \%$ according to Sørensen (1948), while floristic similarity of 5 relevés from the synthetic table of the same association (Krause and Ludwig, ibid.) is slightly higher (31\%), which does not allow for its classification into the same association. In addition, Zupančič et al. (1986: 12) classify the association Erico-Quercetum petraeae into the alliance Quercion robori-petraeae $=$ Quercion roboris and into the order Quercetalia roboripetraeae $=$ Quercetalia roboris, while we classify the studied stands into the order Quercetalia pubescentipetraeae. Floristic dissimilarity is further confirmed by hierarchical classification (Figures 2 and 3).

Based on these comparisons, we decided to classify sessile oak stands from the Vipavska brda into the new association Chamaecytiso hirsuti-Quercetum petraeae ass. nov. hoc loco. Its diagnostic species are Quercus petraea (the edifier of all four compared communities that characterises the newly described association against the primary association Seslerio autumnalis-Fagetum), Erica carnea, Sorbus aria, Sesleria autumnalis, Chamaecytisus hirsutus, Lathyrus linifolius, Loranthus europaeus and Erythronium dens-canis. These species undoubtedly differentiate the studied stands both from floristically slightly similar stands of the associations Seslerio autumnalis-Quercetum petraeae and Melampyro-Quercetum petraeae as well as from physiognomically similar (with dominant Erica carnea in the herb layer) stands of the association Erico-Quercetum petraeae in Bosnia. The differential species that distinguish them from the stands of the association Seslerio-Quercetum are Loranthus europaeus, Lathyrus linifolius and Erythronium dens-canis, partly also Erica carnea and Sorbus aria. The species that differentiate them from the stands of the association Melampyro-Quercetum are Sorbus aria, Sesleria autumnalis, Chamaecytisus hirsutus, Lathyrus linifolius, Erythronium dens-canis and Loranthus europaeus, and the species that differentiate them from the stands of the association Erico-Quercetum are Sorbus aria, Sesleria autumnalis, Lathyrus linifolius and Loranthus europaeus. The diagnostic species characterise the new association both ecologically (Erica carnea, Sorbus aria, Chamaecytisus hirsutus, Lathyrus linifolius, Loranthus europaeus) and phytogeographically (especially Sesleria autumnalis and Erythronium dens-canis). The nomenclature type of the new association, holotypus, is relevé No. 5 in Table 1.

\section{CONCLUSIONS}

\section{ZAKLJUČKI}

Stands of Quercus petraea with Chamaecytisus hirsutus and Erica carnea on shady slopes of the Vipavska 
brda under the Vrhe plateau are an easily recognised degradation stage on potential sites of beech forest from the association Seslerio autumnalis-Fagetum and are also easily distinguishable from other stands. The forest's surface area is estimated at between 500 ha and 1,000 ha (so far, it has been mapped as the association Melampyro vulgati-Quercetum petraeae, Reščič, in litt.). Stands of this stage are mainly distributed in the Karst forest management region, in the forest management unit Vrhe and on a smaller area also in the Ajdovščina forest management unit within the Tolmin forest management region. Progressive development back into the beech forest has been very slow. Now, in the absence of human impact (such as litter gathering), it is hindered by natural factors (convex slopes subject to erosion) and by the fact that due to the warm climate beech in this area occurs on the edge of its natural distribution area. It therefore makes sense to treat these stands at the level of the association Chamaecytiso hirsuti-Quercetum petraeae and to make sure that their role of protection against erosion is considered in forest management practice. They play a significant role also as a biotope, i.e. as a site of the rare hybrid Sorbus latifolia s. lat. (see Avdibegović et al., 2013), protected species Erythronium dens-canis, Platanthera bifolia, $P$. chlorantha, Lilium martagon, Cephalanthera longifolia, Neottia nidus-avis, Sedum maximum (Anon., 2004) and of Leucobryum glaucum, a moss species of conservation concern (Anon., 2002). In terms of floristics and site conditions, the stands of the new association slightly resemble the stands of associations Melampyro vulgate-Quercetum petraeae, Seslerio autumnalis-Quercetum petraeae and Erico carneae-Quercetum petraeae, but also distinctly differ from them in terms of syndynamics, certain ecological factors and in the occurrence of several species. They are characterised by the shady aspect, flysch bedrock, lessivé eutric brown soils, the sub-Mediterranean climate and differential species such as Erica carnea, Sorbus aria, Lathyrus linifolius, Loranthus europaeus and Erythronium dens-canis.

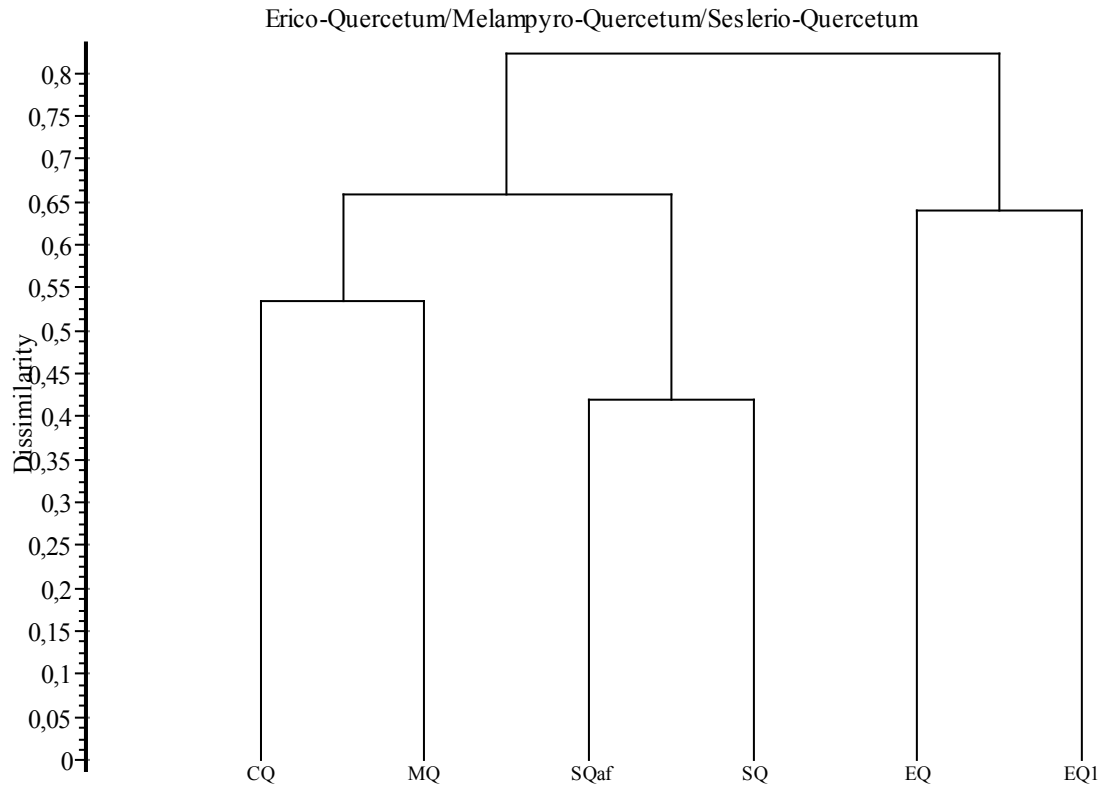

Fig. 2: Dendrogram of stands of the syntaxa Chamaecytiso hirsuti-Quercetum petraeae (CQ), Melampyro-Quercetum petraeae (MQ), Seslerio autumnalis-Quercetum petraeae (SQ), Seslerio autumnalis-Quercetum petraeae avenelletosum flexuosae (SQaf) and Erico-Quercetum petraeae (EQ, EQ1) from southwestern Slovenia and Bosnia, UPGMA, similarity ratio
Slika 2: Dendrogram sestojev sintaksonov Chamaecytiso hirsuti-Quercetum petraeae (CQ), Melampyro-Quercetum petraeae (MQ), Seslerio autumnalis-Quercetum petraeae (SQ), Seslerio autumnalis-Quercetum petraeae avenelletosum flexuosae (SQaf) in Erico-Quercetum petraeae (EQ, EQ1) iz jugovzhodne Slovenije in Bosne, UPGMA, količnik podobnosti »similarity ratio«

CQ Chamaecytiso hirsuti-Quercetum petraeae ass. nov., this article

MQ Melampyro vulgati-Quercetum petraeae Puncer \& Zupančič 1979 var. geogr. Fraxinus ornus (Puncer \& Zupančič) Zupančič 1994 (Puncer in Zupančič, 1979)

SQaf Seslerio autumnalis-Quercetum petraeae Poldini (1964) 1982 avenelletosum flexuosae Poldini 1982 (Poldini, 1982)

SQ Seslerio autumnalis-Quercetum petraeae Poldini (1964) 1982 (Zupančič, 1999)

EQ Erico-Quercetum petraeae Krause et Ludwig ex Horvat 1959 (Ritter-Studnička, 1970)

EQ1 Erico-Quercetum petraeae Krause et Ludwig ex Horvat 1959 (Krause and Ludwig, 1957) 
Classification of the new association into higher syntaxonomical units is as follows:

Class: Querco-Fagetea Br.-Bl. et Vlieger in Vlieger 1937

Order: Quercetalia pubescenti-petraeae Klika 1933

Alliance: Carpinion orientalis Horvat 1958

Suballiance: Seslerio autumnalis-Ostryenion Blasi, Di Pietro \& Filesi 2004 = Ostryo-Carpinenion orientalis Horvat (1954) 1958 nom. illeg.

Association: Chamaecytiso hirsuti-Quercetum petraeae ass. nov. hoc loco

\section{POVZETEK}

\section{SUMMARY}

Po standardni srednjeevropski metodi smo fitocenološko preučili sestoje gradna (Quercus petraea) $\mathrm{v}$ flišnem gričevju Vipavskih brd in na robu planote Vrhe (jugozahodna Slovenija), v katerih v zeliščni plasti prevladuje vrsta Erica carnea (slika 1, preglednica 1). Uspevajo na položnih do strmih osojnih pobočjih, na nadmorski višini od $250 \mathrm{~m}$ do $550 \mathrm{~m}$. Prevladujoči talni tip so evtrična rjava tla, ki so pogosto izprana. Potencialno naravna vegetacija osojnih pobočij tega gričevja je bukov gozd iz asociacije Seslerio autumnalis-
Fagetum, kar potrjujejo nekateri še ohranjeni sestoji, ki smo jih prav tako fitocenološko popisali (preglednica 2). Zdaj prevladujoče sestoje gradna uvrščamo v asociacijo Seslerio autumnalis-Quercetum petraeae. Nizki panjevski hrastovi sestoji s spomladansko reso uspevajo v podobnih rastiščnih razmerah kot sestoji bukve ali gradna $\mathrm{z}$ jesensko vilovino, a imajo očitno različno zeliščno plast. Degradacijo in s tem poslabšanje talnih in posledično rastnih razmer in spremembo vrstne sestave je povzročila erozija (odnašanje prsti, na več krajih jo vidimo še zdaj) in človekova pretekla dejavnost, predvsem steljarjenje. To se kaže v zgornjih horizontih tal, ki so precej kisli ( $\mathrm{pH} \mathrm{H}_{2} \mathrm{O} 4,8, \mathrm{pH} \mathrm{CaCl} \mathrm{3,8} \mathrm{do} \mathrm{4,0),}$ oblika humusa je prhnina (razmerje $\mathrm{C} / \mathrm{N}$ je nad 20). Z globino se lastnosti tal spremenijo in je $\mathrm{pH}$ precej višji. Steljarjenje je eden izmed verjetnih razlogov, ki je posledično pospešil procese izpiranja bazično delujočih kationov (Ca, Mg, K). Vrsta Erica carnea je sicer značilna za bazična tla, vendar lahko dobro uspeva tudi na tleh, ki so v teku razvoja postala kisla. Preučene hrastove sestoje smo primerjali s podobnimi gradnovimi združbami iz asociacij Melampyro vulgati-Quercetum petraeae, Seslerio autumnalis-Quercetum petraeae in Erico carneae-Quercetum petraeae in ugotovili največjo

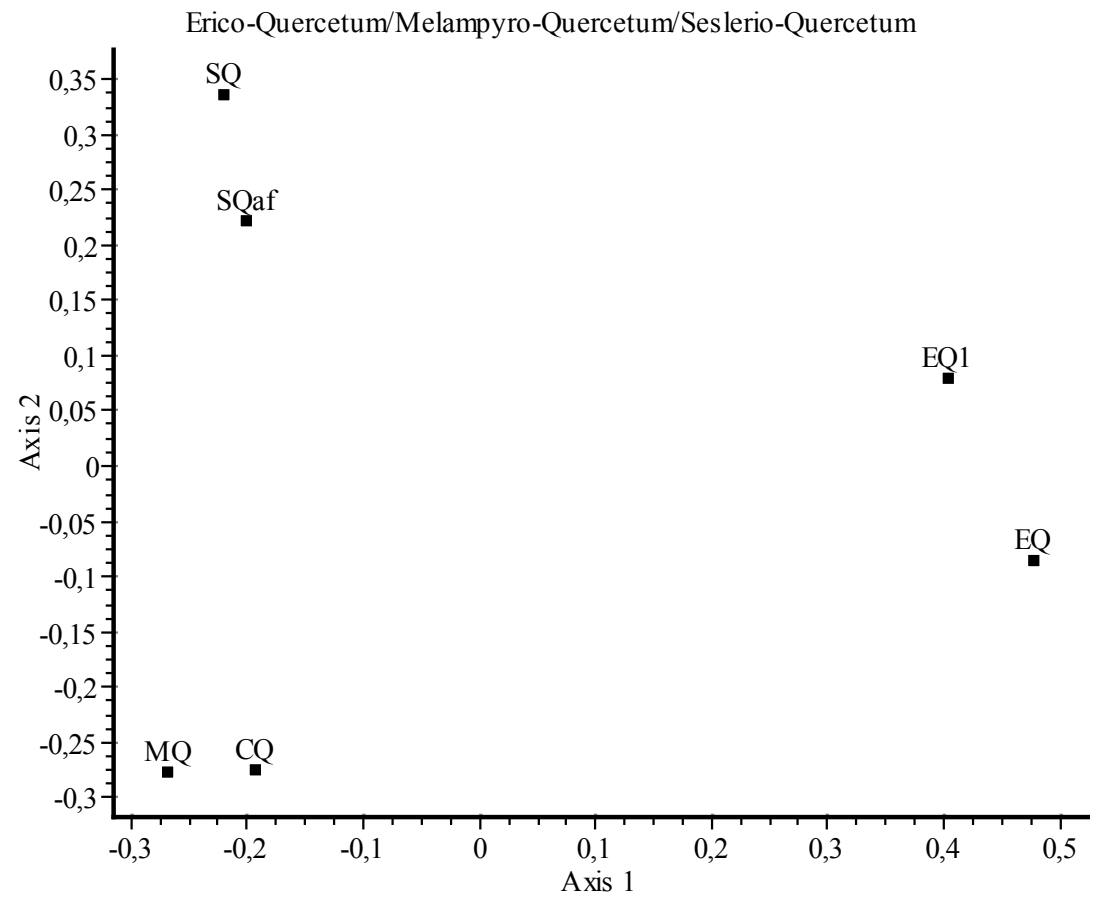

Fig. 3: Two-dimensional scatter diagram of stands of the syntaxa Chamaecytiso hirsuti-Quercetum petraeae (CQ), Melampyro-Quercetum petraeae (MQ), Seslerio autumnalisQuercetum petraeae (SQ), Seslerio autumnalis-Quercetum petraeae avenelletosum flexuosae (SQaf) and Erico-Quercetum petraeae (EQ, EQ1) from southwestern Slovenia and Bosnia, PCoA, similarity ratio
Slika 3: Dvorazsežni ordinacijski diagram sestojev sintaksonov Chamaecytiso hirsuti-Quercetum petraeae (CQ), Melampyro-Quercetum petraeae (MQ), Seslerio autumnalis-Quercetum petraeae (SQ), Seslerio autumnalis-Quercetum petraeae avenelletosum flexuosae (SQaf) in Erico-Quercetum petraeae (EQ, EQ1) iz jugovzhodne Slovenije in Bosne, PCoA, količnik podobnosti »similarity ratio« 
floristično podobnost s sestoji asociacije Melampyro vulgati-Quercetum petraeae iz flišnih Brkinov. Od njih se razlikujejo $\mathrm{v}$ talnih razmerah, sestavi po skupinah diagnostičnih vrst (preglednica 5), po izvirni združbi, ki je v primeru asociacije Melampyro-Quercetum kisloljubno bukovje (Castaneo-Fagetum sylvatice), in po pripadnosti višjim sintaksonomskim enotam (asociacijo Melampyro-Quercetum uvrščamo v zvezo Quercion roboris in v red Quercetalia roboris). Ekološko nekoliko podobni so tudi sestoji subasociacije Seslerio autumnalis-Quercetum petraeae avenelletosum flexuosae, a je njihova floristična sestava že precej drugačna, kar potrjuje hierarhična klasifikacija (sliki 2 in 3). Ta kaže tudi na očitno floristično različnost s sestoji asociacije Erico-Quercetum petraeae, ki so jo opisali v Bosni, na serpentinski podlagi. Ti sestoji imajo kljub podobnosti v dominantnih vrstah drevesne in zeliščne plasti, gradnu in spomladanski resi, drugačno sindinamiko in so povezani z združbami črnega in rdečega bora. $\mathrm{Na}$ podlagi teh primerjav drugotne gradnove sestoje iz Vipavskih brd uvrščamo v novo asociacijo Chamaecytiso hirsuti-Quercetum petraeae ass. nov. hoc loco (zveza Carpinion orientalis, red Quercetalia pubescent-petraeae). Njen nomenklaturni tip, holotypus, je popis št. 5 v preglednici 1. Njene diagnostične vrste so Quercus petraea, Erica carnea, Sorbus aria, Sesleria autumnalis, Chamaecytisus hirsutus, Lathyrus linifolius, Loranthus europaeus in Erythronium dens-canis. Sestoji novo opisane asociacije so lahko prepoznaven in od drugih sestojev lahko ločljiv degradacijski stadij na potencialnih rastiščih bukovega gozda iz asociacije Seslerio autumnalis-Fagetum. Njegova površina ni zanemarljiva in jo ocenjujemo med 500 ha in 1000 ha. Razširjeni so v Kraškem gozdnogospodarskem območju, v gozdnogospodarski enoti Vrhe in na manjši površini tudi v Tolminskem gozdnogospodarskem območju, v gozdnogospodarski enoti Ajdovščina. Progresivni razvoj nazaj v bukov gozd je zelo počasen. Zdaj ko ni več človekovih vplivov (na primer steljarjenja), ga zavirajo naravni dejavniki (izbokla pobočja, izpostavljena eroziji) in dejstvo, da je bukev tu zaradi toplega podnebja na robu svojega naravnega areala.

\section{ACKNOWLEDGEMENTS}

\section{ZAHVALA}

Oak forest research in the Vipava valley was partly conducted within the framework of the target research project Updating of the vegetation system for the forest management planning purposes (V4-1141), funded by the Slovenian Research Agency and Ministry of Agriculture and the Environment. Within the scope of this project, the Slovenian Forestry Institute conducted an analysis of the soil sample. I am sincerely grateful to Mag. Tomaž Prus and Dr. Milan Kobal for their help in the interpretation of soil conditions in the stands of the studied community. Matej Reščič and Klavdijo Čokelj helped me with their advice on the forest cover and the condition of forests on both the Vrhe plateau and in the hills of Vipavska brda. I am also grateful to Dr. Metka Culiberg, Prof. Dr. Andraž Čarni and Prof. Dr. Sead Vojniković for helping me to find and obtain relevant literature sources, as well as to Dr. Lado Kutnar and Dr. Andrej Rozman for valuable improvements of the text. I owe special thanks to Prof. Dr. Jean-Paul Theurillat for the literature he so kindly provided and for his advice on the correct author citation of the association EricoQuercetum petraeae. Iztok Sajko prepared Figure 1 for print. English translation by Andreja Šalamon Verbič.

\section{REFERENCES}

\section{LITERATURA}

Aeschimann D., Lauber K., Moser D. M., Theurillat J.-P. 2004. Flora alpina. Bd. 1,2,3.. Bern, Stuttgart, Wien, Haupt Verlag, $1159+$ $1188+323 p$

Anonymus 2002. Pravilnik o uvrstitvi ogroženih rastlinskih in živalskih vrst v rdeči seznam. Priloga 1: Rdeči seznam praprotnic in semenk (Pteridophyta \& Spermatophyta). Ljubljana, Uradni list RS 82/2002.

Anonymous 2004. Uredba o zavarovanih prosto živečih rastlinskih vrstah. Ljubljana, Uradni list RS 46/2004.

Avdibegović M., Vojniković S., Bogunić F., Hajrudinović A., Dautbašić M., Brajić A., Balić B., Delić S., Mutabdžija S., Marić B., Bećirović D. 2013. Razvoj regulatornih instrumenata šumarske politike. Izdvajanje šuma visoke zašitne vrijednosti (HCVF) na području Š.P.P »Igmansko«. Sarajevo, Šumarski fakultet Univerze u Sarajevu: $78 \mathrm{p}$.

Beus V. 1997. Fitocenologija za 2. razred šumarske tehničke škole. Sarajevo, Federacija Bosne i Hercegovine. Ministarstvo obrazovanja, nauke, kulture i sporta i "Sarajevo-Publishing": 138 p.

Braun-Blanquet J. 1964. Pflanzensoziologie. Grundzüge der Vegetationskunde. 3. Auf., Wien-New York, Springer Verlag: 865 p.

Buser S. 1973. Osnovna geološka karta SFRJ 1:100 000. Tolmač lista Gorica. Beograd, Zvezni geološki zavod: 50 p.

Buser S. 2009. Geološka karta Slovenije 1: 250.000. Geological map of Slovenia 1.250,000. Ljubljana, Geološki zavod Slovenije.

Cegnar T. 1998. Temperatura zraka. In: Fridl J., Kladnik D., Orožen Adamič M., Perko D. (eds.): Geografski atlas Slovenije. Država v prostoru in času. Ljubljana, Državna založba Slovenije: 100-101.

Čarni A., Marinček L., Seliškar A., Zupančič M. 2002. Vegetacijska karta gozdnih združb Slovenije 1: 400.000. Ljubljana, Biološki inštitut Jovana Hadžija, ZRC SAZU.

Dakskobler I. 1997. Geografske variante asociacije Seslerio autumnalis-Fagetum (Ht.) M. Wraber ex Borhidi 1963. Razprave 4. razreda SAZU (Ljubljana), 38, 8: 165-255.

Fabijanić B., Fukarek P., Stefanović V. 1963. Pregled osnovnih tipova šumske vegetacije. Lepenica. Posebna izdanja Naučnog društva SR Bosne i Hercegovine (Sarajevo) 3: 85-129.

Horvat I. 1959. Sistematski odnosi termofilnih hrastovih i borovih šuma Jugoistočne Europe. Biol. glas. Hrv. prirod. društva (Zagreb) 12: 1-40.

Horvat I., Glavač V., Ellenberg H. 1974. Vegetation Südosteuropas. Stuttgart,Gustav Fischer Verlag: 768 p. 
Kladnik D., 2000. Vipavska brda. In: Voglar D. (ed.): Enciklopedija Slovenije 14 U/We, Ljubljana, Mladinska knjiga, p. 257.

Lovrenčak F. 1998. Prsti. In: Fridl J., Kladnik D., Orožen Adamič M., Perko D. (eds.): Geografski atlas Slovenije. Država v prostoru in času. Ljubljana, Državna založba Slovenije: 114-115.

Krause W., Ludwig, W. 1957. Zur Kenntnis der Flora und Vegetation auf Serpentinstandorten des Balkans. 2. Pflanzengesellschaften und Standorte im Gostović-Gebiet (Bosnien). Flora (Regensburg) 145: 78-131.

Maarel van der E. 1979. Transformation of cover-abundance values in phytosociology and its effects on community similarity. Vegetatio 39, 2: 97-114.

Martinčič A. 2003. Seznam listnatih mahov (Bryopsida) Slovenije. Hacquetia (Ljubljana) 2, 1: 91-166.

Martinčič, A. 2011. Seznam jetrenjakov (Marchanthiophyta) in rogovnjakov (Anthocerotophyta) Slovenije. Annotated Checklist of Slovenian Liverworts (Marchanthiophyta) and Hornworts (Anthocerotophyta). Scopolia (Ljubljana) 72: 1-38.

Martinčič A., Wraber T., Jogan N., Podobnik A., Turk B., Vreš B., Ravnik V., Frajman B., Strgulc Krajšek S., Trčak B., Bačič T., Fischer M. A., Eler K.,Surina B. 2007. Mala flora Slovenije. Ključ za določanje praprotnic in semenk. Četrta, dopolnjena in spremenjena izdaja. Ljubljana,Tehniška založba Slovenije: 967 p.

Podani J. 2001. SYN-TAX 2000. Computer Programs for Data Analysis in Ecology and Systematics. User's Manual, Budapest: 53 p.

Poldini L. 1982. Ostrya carpinifolia-reiche Wälder und Gebüsche von Friaul-Julisch-Venezien (NO-Italien) und Nachbargebieten. Studia Geobotanica (Trieste) 2: 69-122.

Poldini L. 1988. Übersicht des Verbandes Ostryo-Carpinion orientalis (Quercetalia pubescentis) in SO-Europa. Phytocoenologia (Stuttgart-Braunschweig),16, 1: 125-143.

Poldini L. 1989. La vegetazione del Carso isontino e triestino. Trieste, Ed. Lint, 313 p.

Puncer I., Zupančič M. 1979. Novi združbi gradna v Sloveniji (Melampyro vulgati-Quercetum petraeae ass. nova s. lat.). Scopolia (Ljubljana) 2: 1-47 + fitocenološke tabele.
Rajšp V., Trpin D. 1997. Slovenija na vojaškem zemljevidu $1763-$ 1787 (1804). Opisi in karte, 3. zvezek. Ljubljana, ZRC SAZU in Arhiv Republike Slovenije: $435 \mathrm{p}$.

Ritter-Studnička H. 1970. Die Vegetation der Serpentinvorkommen in Bosnien. Vegetatio 21: 75-156.

Seliškar T., Vreš B., Seliškar A. 2003. FloVegSi 2.0. Računalniški program za urejanje in analizo bioloških podatkov. Ljubljana, Biološki inštitut ZRC SAZU.

Sørensen Th. 1948. A method of establishing groups of equal amplitude in plant sociology based on similarity of species content. Det Kongelige Danske Videnskaberns Selskab, Biologiske Skrifter (København) 5, 4:1-34.

Stefanović V. 1984. Cenohorološki odnosi kitnjakovih šuma (Quercetum petraeae sens. Lat.) u Bosni i Hercegovini. Bilten Društva ekologa Bosne i Hercegovine (Sarajevo), Serija B, Br. 2, Knj. 1: 203-210.

Šilc U., Čarni A. 2012. Conspectus of vegetation syntaxa in Slovenia. Hacquetia (Ljubljana) 11, 1: 113-164.

Urbančič M., Simončič P., Prus T., Kutnar L. 2005. Atlas gozdnih tal. Ljubljana, Zveza gozdarskih društev Slovenije, Gozdarski vestnik \& Gozdarski inštitut Slovenije: 100 str.

Zupančič B. 1998. Padavine. In: Fridl J., Kladnik D., Orožen Adamič M., Perko D. (eds.): Geografski atlas Slovenije. Država v prostoru in času. Ljubljana, Državna založba Slovenije: 98-99.

Zupančič M. 1994. Popravki imen nekaterih rastlinskih združb v luči novega kodeksa. Hladnikia (Ljubljana) 2: 33-40.

Zupančič M. 1999. Novosti o gozdno-grmiščni vegetaciji slovenskega submediterana. Razprave 4. razreda SAZU (Ljubljana) 40, 8: 195-313.

Zupančič M., Jovanović, B., Lakušić, R., Rizovski R., Trinajstić I. (eds.) 1986. Prodromus Phytocoenosum Jugoslaviae ad mappam vegetationis m 1:200 000. Bribir-Ilok, Naučno veće vegetacijske karte Jugoslavije: 46 p. 
Table 1: Chamaecytiso hirsuti-Quercetum petraeae ass. nov. hoc loco
Preglednica 1: Chamaecytiso hirsuti-Quercetum petraeae ass. nov. hoc loco

\begin{tabular}{|c|c|c|c|c|c|c|c|c|c|c|c|c|c|c|c|c|c|c|c|c|c|c|c|c|c|c|c|c|c|}
\hline \multicolumn{2}{|c|}{\begin{tabular}{|l|}
$\begin{array}{l}\text { Number of relevé (Zaporedna } \\
\text { številka popisa) }\end{array}$ \\
\end{tabular}} & 1 & 2 & 3 & 4 & 5 & 6 & 7 & 8 & 9 & 10 & 11 & 12 & 13 & 14 & 15 & 16 & 17 & 18 & 19 & 20 & 21 & 22 & 23 & 24 & 25 & 26 & Pr. & Fr. \\
\hline \multicolumn{2}{|l|}{$\begin{array}{l}\text { Database number of relevé } \\
\text { (Delovna številka popisa) }\end{array}$} & 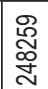 & 兽 & $\underset{\substack{N \\
\sim N}}{\underset{N}{N}}$ & \begin{tabular}{|l|} 
\\
$\stackrel{\infty}{\infty}$ \\
$\stackrel{\infty}{\sim}$ \\
\multirow{2}{*}{}
\end{tabular} & 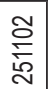 & 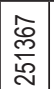 & 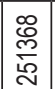 & 总 & 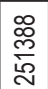 & \begin{tabular}{|l|}
$\stackrel{\mathscr{m}}{\mathrm{m}}$ \\
$\stackrel{\mathrm{m}}{\mathrm{N}}$ \\
\end{tabular} & 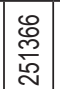 & 票 & 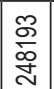 & 齐 & 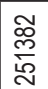 & 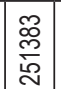 & 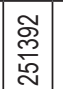 & 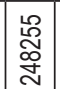 & 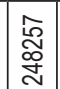 & 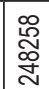 & 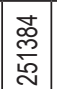 & 䁶 & 恕 & 胥 & \begin{tabular}{|l|} 
品 \\
岕 \\
\end{tabular} & 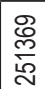 & & \\
\hline $\begin{array}{l}\text { Elevation in } \mathrm{m} \text { (Nadmor- } \\
\text { ska višina v } \mathrm{m} \text { ) }\end{array}$ & & 410 & 305 & 400 & 290 & 360 & 415 & 360 & 535 & 425 & 455 & 500 & 340 & 390 & 310 & 335 & 380 & 395 & 320 & 460 & 440 & 410 & 420 & 450 & 375 & 455 & 315 & & \\
\hline Aspect (Lega) & & $E$ & $\mathrm{NE}$ & $\mathrm{N}$ & $E$ & $E$ & SE & SE & $\mathrm{NE}$ & $N$ & $\mathrm{~N}$ & NEE & NE & $\mathrm{NE}$ & $\mathrm{N}$ & NNE & $\mathrm{N}$ & NE $\wedge$ & NNE & NE & $\mathrm{NE}$ & $\mathrm{NE}$ & SE & $\mathrm{NE}$ & $\mathrm{NE}$ & $\mathrm{NE}$ & SEE & & \\
\hline \begin{tabular}{|l|} 
Slope in degrees (Nagib v \\
stopinjah)
\end{tabular} & & 25 & 5 & 25 & 25 & 25 & 25 & 25 & 20 & 15 & 15 & 25 & 20 & 20 & 25 & 25 & 20 & 15 & 25 & 10 & 15 & 10 & 15 & 15 & 20 & 15 & 30 & & \\
\hline $\begin{array}{l}\text { Parent material (Matična } \\
\text { podlaga) }\end{array}$ & & Fly & Fly & Fly & Fly & Fly & Fly & Fly & Fly & Fly & Fly & Fly & Fly & Fly & Fly & Fly & Fly & Fly & Fly & Fly & Fly & Fly & Fly & Fly & Fly & Fly & Fly & & \\
\hline Soil (Tla) & & Eu & Eu & $\mathrm{Eu}$ & $\mathrm{Eu}$ & $\mathrm{Eu}$ & $\mathrm{Eu}$ & $\mathrm{Eu}$ & $\mathrm{Eu}$ & Eu & $\mathrm{Eu}$ & $\mathrm{Eu}$ & $\mathrm{Eu}$ & $\mathrm{Eu}$ & Eu & Eu & $\mathrm{Eu}$ & Eu & Eu & Eu & Eu & Eu & $\mathrm{Eu}$ & Eu & $\mathrm{Eu}$ & $\mathrm{Eu}$ & Eu & & \\
\hline \begin{tabular}{|l|} 
Stoniness in \% (Kamnitost \\
$\mathrm{v} \%$ )
\end{tabular} & & 10 & 0 & 0 & 10 & 0 & 0 & 5 & 0 & 0 & 0 & 0 & 1 & 5 & 0 & 5 & 1 & 5 & 0 & 0 & 0 & 0 & 0 & 5 & 0 & 0 & 5 & & \\
\hline \multicolumn{30}{|l|}{$\begin{array}{l}\text { Cover in \% (Zastiranje } \\
\text { v \%): }\end{array}$} \\
\hline $\begin{array}{l}\text { Upper tree layer (Zgornja } \\
\text { drevesna plast) }\end{array}$ & E3b & 80 & 80 & 70 & 80 & 80 & 80 & 70 & 80 & 80 & 80 & 80 & 90 & 90 & 80 & 80 & 80 & 80 & 80 & 80 & 80 & 80 & 90 & 90 & 80 & 90 & 80 & & \\
\hline \begin{tabular}{|l|} 
Lower tree layer (Spodnja \\
drevesna plast)
\end{tabular} & E3a & 5 & 10 & 20 & 10 & 10 & 10 & 20 & 10 & 5 & 5 & 10 & 5 & 5 & 5 & 10 & 5 & 5 & 0 & 5 & 5 & 5 & 0 & 5 & 10 & 0 & 5 & & \\
\hline $\begin{array}{l}\text { Shrub layer (Grmovna } \\
\text { plast) }\end{array}$ & E2 & 20 & 30 & 50 & 10 & 20 & 20 & 30 & 20 & 10 & 20 & 20 & 10 & 20 & 20 & 25 & 15 & 30 & 20 & 20 & 20 & 20 & 20 & 20 & 20 & 10 & 10 & & \\
\hline Herb layer (Zeliščna plast) & E1 & 90 & 90 & 90 & 70 & 90 & 95 & 90 & 100 & 95 & 100 & 100 & 80 & 80 & 95 & 90 & 95 & 95 & 90 & 90 & 95 & 95 & 95 & 95 & 95 & 100 & 90 & & \\
\hline $\begin{array}{l}\text { Moss layer (Mahovna } \\
\text { plast) }\end{array}$ & E0 & 10 & 10 & 1 & 20 & 5 & 5 & 5 & 5 & 5 & 5 & 5 & 30 & 30 & 5 & 10 & 10 & 5 & 10 & 10 & 5 & 10 & 5 & 5 & 5 & 5 & 5 & & \\
\hline \begin{tabular}{|l|} 
Maximum diameter of tre- \\
es (Največji prsni premer \\
dreves)
\end{tabular} & $\mathrm{cm}$ & 30 & 30 & 25 & 30 & 35 & 35 & 30 & 25 & 25 & 25 & 30 & 30 & 30 & 25 & 25 & 35 & 30 & 30 & 25 & 25 & 25 & 25 & 25 & 30 & 25 & 30 & & \\
\hline \begin{tabular}{|l|} 
Maximum height of tress \\
(Največja drevesna višina)
\end{tabular} & $\mathrm{m}$ & 15 & 17 & 13 & 17 & 16 & 14 & 15 & 10 & 14 & 12 & 14 & 15 & 16 & 14 & 15 & 15 & 12 & 15 & 15 & 14 & 12 & 12 & 12 & 14 & 9 & 15 & & \\
\hline $\begin{array}{l}\text { Number of species (Šte- } \\
\text { vilo vrst) }\end{array}$ & & 32 & 49 & 21 & 38 & 28 & 24 & 30 & 26 & 32 & 28 & 34 & 39 & 28 & 36 & 39 & 37 & 28 & 37 & 28 & 26 & 26 & 26 & 20 & 26 & 23 & 38 & & \\
\hline $\begin{array}{l}\text { Relevé area (Velikost } \\
\text { popisne ploskve) }\end{array}$ & $\mathrm{m}^{2}$ & 400 & 200 & 400 & 200 & 400 & 400 & 400 & 400 & 200 & 200 & 400 & 200 & 200 & 200 & 400 & 400 & 200 & 400 & 400 & 400 & 400 & 200 & 200 & 200 & 200 & 400 & & \\
\hline $\begin{array}{l}\text { Date of taking relevé } \\
\text { (Datum popisa) }\end{array}$ & & 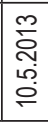 & 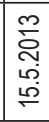 & 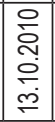 & 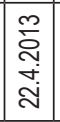 & 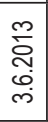 & 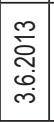 & 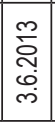 & 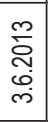 & 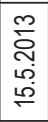 & 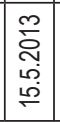 & 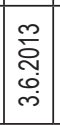 & 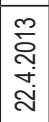 & 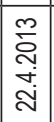 & 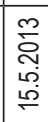 & 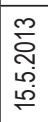 & 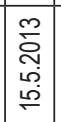 & 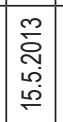 & 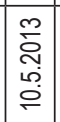 & 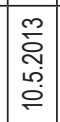 & 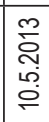 & 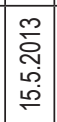 & 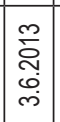 & 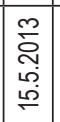 & 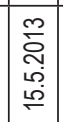 & 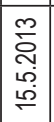 & 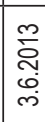 & & \\
\hline Locality (Nahajališče) & & 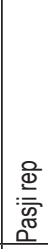 & 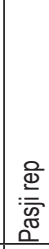 & 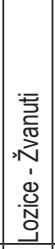 & 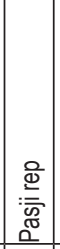 & 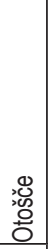 & 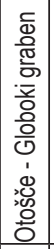 & 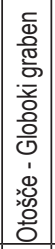 & 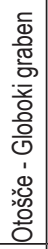 & 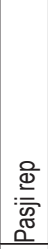 & 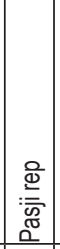 & 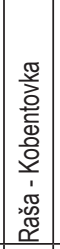 & 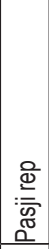 & 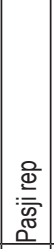 & 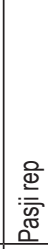 & 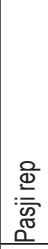 & 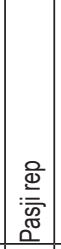 & 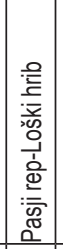 & 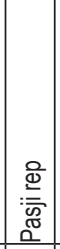 & 월 & 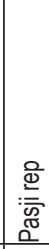 & $\begin{array}{c}\stackrel{0}{\frac{0}{2}} \\
: \bar{c} \\
\alpha \\
\alpha\end{array}$ & 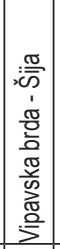 & 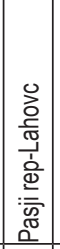 & 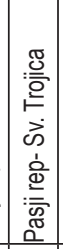 & 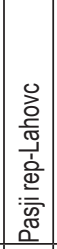 & 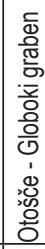 & & \\
\hline Quadrant (Kvadrant) & & $\frac{d}{d}$ & $\frac{d}{d}$ & 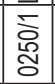 & 竞 & 容 & 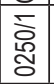 & 荾 & 总 & 总 & 空 & 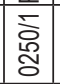 & 总 & 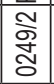 & 竞 & 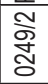 & 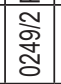 & 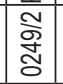 & 学 & 竞 & 竞 & 竞 & 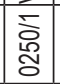 & 空 & 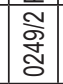 & 产 & 空 & & \\
\hline Coordinate GK Y (D-48) & $\mathrm{m}$ & 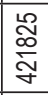 & $\underset{\substack{m \\
\sim}}{\stackrel{\infty}{q}}$ & 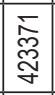 & 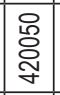 & 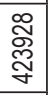 & 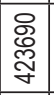 & \begin{tabular}{|l|}
$\underset{o}{J}$ \\
$\underset{\sim}{\sim}$ \\
\end{tabular} & 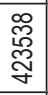 & 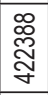 & 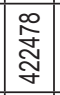 & 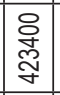 & \begin{tabular}{|l}
\multirow{2}{*}{} \\
ণ్ \\
צे
\end{tabular} & \begin{tabular}{|l|}
\multirow{2}{*}{ 定 } \\
\end{tabular} & 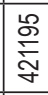 & 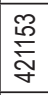 & 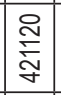 & $\underset{\bar{\sigma}}{\stackrel{\check{\sigma}}{\Im}}$ & 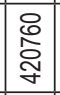 & \begin{tabular}{|c|}
$\stackrel{\widetilde{m}}{\sim}$ \\
$\stackrel{\sim}{\sim}$
\end{tabular} & 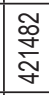 & 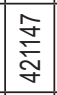 & 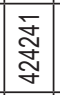 & 志 & \begin{tabular}{|l|}
$\frac{10}{m}$ \\
$\stackrel{y}{\Im}$ \\
\end{tabular} & 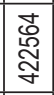 & 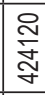 & & \\
\hline Coordinate GK X (D-48) & $\mathrm{m}$ & 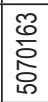 & \begin{tabular}{|l|}
0 \\
08 \\
0 \\
0 \\
\end{tabular} & 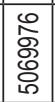 & 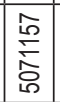 & 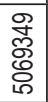 & 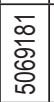 & 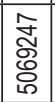 & 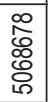 & 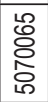 & 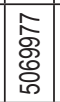 & 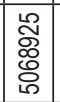 & \begin{tabular}{|l|}
\multirow{2}{0}{} \\
응 \\
\&.
\end{tabular} & 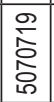 & $\begin{array}{l}\text { o } \\
\text { o } \\
\text { 今. } \\
\text { of }\end{array}$ & 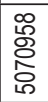 & \begin{tabular}{|l|}
$\overline{8}$ \\
$o$ \\
今. \\
$口$
\end{tabular} & 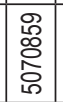 & 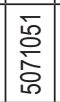 & 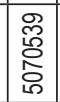 & \begin{tabular}{|l|}
0 \\
0 \\
0 \\
0
\end{tabular} & 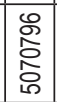 & \begin{tabular}{|l|} 
\\
\\
$\delta$ \\
0 \\
0 \\
0
\end{tabular} & 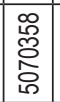 & 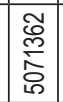 & 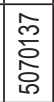 & 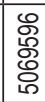 & & \\
\hline
\end{tabular}

\begin{tabular}{|c|c|c|c|c|c|c|c|c|c|c|c|c|c|c|c|c|c|c|c|c|c|c|c|c|c|c|c|c|c|c|}
\hline \multirow[b]{2}{*}{ QR } & \multicolumn{28}{|c|}{ Diagnostic species of the association (Diagnostične vrste asociacije) } & \multirow[b]{2}{*}{26} & \multirow[b]{2}{*}{100} \\
\hline & Quercus petraea & E3b & 5 & 5 & 5 & 5 & 5 & 5 & 5 & 5 & 5 & 5 & 5 & 5 & 5 & 5 & 5 & 5 & 4 & 5 & 5 & 5 & 5 & 5 & 5 & 5 & 5 & 5 & & \\
\hline QR & Quercus & $3 a$ & & + & & + & 1 & + & 1 & & & + & + & + & + & + & & + & & & + & + & + & & & & & 1 & 15 & 58 \\
\hline QR & Quercus petraea & $E 2 b$ & . & & & & + & & . & + & & + & & + & . & + & + & & + & & . & . & & & 1 & & . & . & 8 & 31 \\
\hline$Q R$ & Quercus petraea & E2a & & & & & + & & 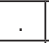 & & + & + & & & . & + & & & & & . & & & & & & + & 1 & 6 & 23 \\
\hline$Q R$ & Quercus petrae & E1 & 1 & 1 & 1 & 1 & 1 & + & 1 & + & 1 & & 1 & 1 & 1 & 1 & $\begin{array}{l}T \\
\end{array}$ & + & + & + & 1 & + & & 1 & 1 & + & 1 & & 23 & 88 \\
\hline EP & Erica c & E1 & 4 & 3 & 5 & 4 & 5 & 5 & 5 & 5 & 5 & 4 & 5 & r & 4 & 5 & 4 & 4 & 5 & 4 & 4 & 5 & 5 & 5 & 5 & 5 & 5 & 5 & 26 & 100 \\
\hline $\mathrm{QP}$ & Sorbus aria & E3 & + & & + & 1 & + & & + & + & + & + & + & & + & & + & + & + & 1 & + & + & + & + & + & & + & + & 21 & 81 \\
\hline $\mathrm{QP}$ & Sorbus a & $E 2 b$ & 1 & + & & 1 & + & + & 1 & 1 & + & 1 & + & 2 & 2 & 1 & 1 & 1 & + & 1 & + & + & 1 & + & 1 & + & & 1 & 24 & 92 \\
\hline $\mathrm{QP}$ & Sorbus aria & E2a & 1 & 1 & & + & + & 1 & . & + & 1 & & + & 1 & + & 1 & & 1 & + & 1 & + & 1 & 1 & . & + & + & 1 & $\dot{.}$ & 20 & 77 \\
\hline $\mathrm{QP}$ & Sorbus aria & E1 & & & & & & & & + & + & + & & + & + & + & & + & + & & + & 1 & + & 1 & + & + & & + & 15 & 58 \\
\hline $\mathrm{CO}$ & Sesleria autumnalis & E1 & 2 & 2 & + & + & 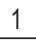 & + & 2 & & + & + & 1 & + & + & + & $\mathrm{L}^{1}$ & . & + & 1 & . & . & + & 2 & + & + & + & + & 22 & 85 \\
\hline EP & Chamaecytisus hirs & E1 & + & + & + & . & + & + & + & $\dot{.}$ & + & + & 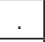 & & 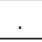 & + & + & + & + & 1 & + & + & 1 & 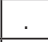 & + & + & & + & 19 & 73 \\
\hline$Q R$ & Lathyrus linifolius & E1 & + & 1 & & + & & & . & + & + & + & + & 1 & + & 1 & 1 & 1 & + & & + & & + & + & & + & & + & 18 & 69 \\
\hline
\end{tabular}




\begin{tabular}{|c|c|c|c|c|c|c|c|c|c|c|c|c|c|c|c|c|c|c|c|c|c|c|c|c|c|c|c|c|c|c|}
\hline & \multicolumn{2}{|c|}{$\begin{array}{l}\text { Number of relevé (Zaporedna } \\
\text { številka popisa) }\end{array}$} & \multirow[t]{2}{*}{1} & \multirow{2}{*}{$\begin{array}{l}2 \\
+\end{array}$} & 3 & 4 & 5 & 6 & 7 & 8 & 9 & 10 & 11 & 12 & 13 & 14 & 15 & 16 & 17 & 18 & 19 & 20 & 21 & 22 & 23 & 24 & 25 & 26 & Pr. & Fr. \\
\hline QP & Loranthus europaeus & E3a & & & & . & + & + & + & + & & & & $r$ & + & $r$ & + & + & & + & + & + & + & + & & . & & + & 16 & 62 \\
\hline EC & Erythronium dens-canis & E1 & & + & + & & + & . & . & 1 & 1 & 1 & & . & 1 & & + & 1 & & 1 & 1 & + & & & & & & + & 13 & 50 \\
\hline QP & Quercetalia pubescenti- & etraea & & & & & & & & & & & & & & & & & & & & & & & & & & & & \\
\hline & Fraxinus ornus & E3 & . & & + & . & + & + & . & + & . & + & &. & . & + & + & & + &. & & & . & + & &. & & + & 10 & 38 \\
\hline & Fraxinus ornus & $E 2 b$ & + & 2 & 1 & 1 & 1 & 1 & 2 & + & 1 & 1 & 1 & 2 & 1 & 2 & 2 & 1 & 1 & 1 & + & + & 1 & 1 & 1 & 2 & 1 & 1 & 26 & 100 \\
\hline & Fraxinus ornus & E2a & 1 & 1 & . & 1 & 2 & 1 & 2 & & \begin{tabular}{|l|}
1 \\
\end{tabular} & 1 & 1 & 1 & 2 & 1 & & 1 & & \begin{tabular}{|l|}
1 \\
\end{tabular} & 2 & 1 & 1 & 1 & 1 & \begin{tabular}{|l|}
1 \\
\end{tabular} & + & 2 & 20 & 77 \\
\hline & Fraxinus ornus & E1 & + & 1 & & + & 1 & . & 1 & & 1 & & 1 & . & . & & + & & & + & 1 & & 1 & 1 & 1 & 1 & 1 & 1 & 16 & 62 \\
\hline & Convallaria majalis & E1 & . & & + & $r$ & + & + & + & 3 & 3 & 3 & 2 & 1 & 1 & + & 3 & 3 & 3 & 3 & 2 & 1 & 1 & 3 & 3 & 3 & 2 & 1 & 24 & 92 \\
\hline & Sorbus torminalis & E3a & . & . & + & . & . & . & + & & . & . & 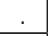 & . &. & & . & . & & 1 & . & 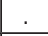 & + & 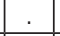 & . & . & & . & 4 & 15 \\
\hline & Sorbus torminalis & $E 2 b$ & + & & + & + & + & + & 1 & & + & . & + & . & . & + & + & + & 2 & 1 & + & + & 1 & + & . & + & & 1 & 19 & 73 \\
\hline & Sorbus torminalis & E2a & + & + & + & . & + & + & 1 & & + & + & 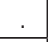 &. &. & + & + & + & 2 & \begin{tabular}{|l|}
1 \\
\end{tabular} & 1 & \begin{tabular}{|l|}
1 \\
\end{tabular} & + & . & . & + & & + & 18 & 69 \\
\hline & Sorbus torminalis & E1 & + & 2 & . & . &. & + & 1 & t & . & + & + & + & + & &. & + & 1 &. & 2 & 1 & 1 & + & . &. & . & + & 15 & 58 \\
\hline & Tanacetum corymbosum & E1 & + & + & . & . & . & . & + & t &. & . & + & + &. & + & + & + & + & + & + & + & + & + & . & + & & . & 15 & 58 \\
\hline & Lathyrus niger & E1 & . & + & & + & & . & + & & . & . & & . &. & &. & . & $r$ & + &. & & . & . & . & + & & . & 6 & 23 \\
\hline & Ostrya carpinifolia & E3 & . & . & . & . & + &. &. & . &. & . & . &. &. & & $r$ & . & - &. &. & $\dot{\square}$ & . & . & . &. & . & + & 3 & 12 \\
\hline & Ostrya carpinifolia & E2 & + & . & . & . & . &. & . & & . & . & . & $r$ & . & &. & . & 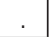 & . & . &. & . & . & . & . & . & . & 2 & 8 \\
\hline & Campanula persicifolia & E1 & . & + & & . & & . &. & & . & . & & . &. & &. & 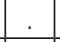 & & + &. & & . & . & . &. & & . & 2 & 8 \\
\hline & Sorbus austriaca s. lat. & E3a & . & & . & . & . & . & . & & . & . & + & . &. & &. & . & & . &. & . & . & 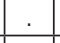 & . & . & . & . & 1 & 4 \\
\hline & Sorbus austriaca s. lat. & $E 2 b$ & + & & & . & & & + & & . & . & &. &. & & $\cdot$ & . & & . & . & & . & & . & . & & & 2 & 8 \\
\hline & Sorbus austriaca s. lat. & E1 & + & & & & & & . & & & . & & . & . & & . & & & . & . & & . & & & . & & & 1 & 4 \\
\hline & Sorbus latifolia s. lat. & E2 & . & & & . & 1 & . &. & & . & . & &. &. & &. & . & & . & . & & . & . & & . & & . & 1 & 4 \\
\hline & Sorbus latifolia s. lat. & E1 & & & & & + & & . & & . & . & & . & . & &. & . & & . & . & & . & & & . & & & 1 & 4 \\
\hline & Sorbus graeca & E2 & & & & & & & . & & & $\dot{.}$ & & . & . & + & & & & . & & & . & & & . & & & 1 & 4 \\
\hline$Q R$ & Quercetalia roboris, Cal & uno-Ul & icete & a, Mo & olinie & & & & & & & & & & & & & & & & & & & & & & & & & \\
\hline & Hieracium sabaudum & E1 & 2 & 1 & + & + & & + & + & + & 1 & + & 1 & + & 1 & 1 & 1 & 1 & 1 & 1 & 1 & 1 & 1 & + & 1 & 1 & + & + & 25 & 96 \\
\hline & Serratula tinctoria & E1 & 1 & & + & + & & &. & & 1 & + & 1 & 1 & 1 & 2 & 2 & 2 & 3 & 2 & 2 & \begin{tabular}{|l|}
1 \\
\end{tabular} & 2 & + & \begin{tabular}{|l|}
1 \\
\end{tabular} & \begin{tabular}{|l|}
1 \\
\end{tabular} & \begin{tabular}{|l|}
1 \\
\end{tabular} & 2 & 21 & 81 \\
\hline & Hieracium racemosum & E1 & . & 1 & + & 1 & 1 & . & 1 & & + & + & + & 1 & . & + & + & . & . & + & + & . & + & + & . & + & + & 1 & 18 & 69 \\
\hline & Genista pilosa & E1 & + & & + & + & + & 1 & + & & + & + & &. & + & + &. & + & + &. & + & 1 & + & & . & + & + & . & 17 & 65 \\
\hline & Castanea sativa & E3a & . & & & 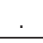 & & . &. & & & . & & . &. & & . & $r$ & + &. & . & & . & . & . &. & & . & 2 & 8 \\
\hline & Castanea sativa & $E 2 b$ & . & . & + & + & . &. & . & . & . & . & . & 1 &. & . & + & + & + &. & . & . & . & . & . & + & . & + & 8 & 31 \\
\hline & Castanea sativa & E2a & . & + & + & $r$ & & . & + & & . & . & & + & . & + & + & + & + &. & $r$ &. & . & & . & + & & + & 12 & 46 \\
\hline & Castanea sativa & E1 & . & + & & + & &. & . & & . & . & &. &. & & . & . & + &. &. & . & + & & . & + & & . & 5 & 19 \\
\hline & Potentilla erecta & E1 & . & + & . & + & . &. & . & + & + & + & . & . & . & 1 & 1 & 1 & + & + &. & . & . & . & $\dot{.}$ & . & + & . & 11 & 42 \\
\hline & Pteridium aquilinum & E1 & . & + & . & 1 & . & 1 & . & & . & . & . & 1 & 1 & 3 & 3 & 2 & 1 & . & . & + & + & & . & . & & 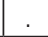 & 11 & 42 \\
\hline CU & Calluna vulgaris & E1 & . & & + & . & + & + & . & & + & . & + &. &. & &. & . & . &. &. & + & + & . & . &. & + & . & 8 & 31 \\
\hline & Chamaecytisus supinus & E1 & . & $\dot{r}$ & . & + & . & . &. & . & . & . & . & + & + & &. & . & + & + &. & . & . & . & . &. & . &. & 5 & 19 \\
\hline & $\begin{array}{l}\text { Melampyrum pratense } \\
\text { subsp. vulgatum }\end{array}$ & E1 & . & + & . & . & . & . & . & 1 & . & . & + & . & . & & . & + & & . & . & . & . & . & . & . & & . & 4 & 15 \\
\hline & Hieracium lachenalii & E1 & + & & & + & & . &. & & . & . & &. &. & & . & $\dot{0}$ & &. &. & + & . & & &. & & & 3 & 12 \\
\hline $\mathrm{MO}$ & Laserpitium prutenicum & E1 & . & . & . & . & . & . & . & & . & . & . &. & . & + & . & . & + &. &. & 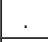 & . & . & . &. & & . & 2 & 8 \\
\hline $\mathrm{CU}$ & Genista germanica & E1 & . & + & & . & & . & . & & . & 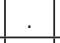 & & . &. & &. & & & . &. & & . & & & . & & . & 1 & 4 \\
\hline & Betonica officinalis & E1 & . & + & & . & & . &. & & . & . & &. &. & &. & . & &. &. & & . & & &. & & & 1 & 4 \\
\hline & Betula pendula & E3b & . &. & & . & & . &. & & $r$ & . & &. &. & &. & . & &. &. & 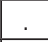 & . & . & . &. & & . & 1 & 4 \\
\hline CU & Phyteuma zahlbruckneri & E1 & . & & & . & & . & . & & . & . & & . & . & & . &. & & . & . & & . & . & & . & & + & 1 & 4 \\
\hline FS & Fagetalia sylvaticae & & & & & & & & & & & & & & & & & & & & & & & & & & & & & \\
\hline & Fagus sylvatica & E3b & 1 & & & $r$ & + & + & $r$ & + & 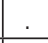 & . & $r$ &. &. & & + & $r$ & &. &. & . & . & . & . &. & &. & 9 & 35 \\
\hline & Fagus sylvatica & E3a & + & + & t & . & 1 & + & $\mathrm{r}$ & + & $r$ & $r$ & + & . & $r$ & &. & . & & . & + & . & . & + & & . & & + & 14 & 54 \\
\hline & Fagus sylvatica & $E 2 b$ & + & . & . & . & 1 & . & + & + & . & . & . & + & + & & + & . & &. &. & . & . & + & . &. & & + & 9 & 35 \\
\hline & Fagus sylvatica & E2a & . & & & $r$ & + & + & . & + & . & + & + & . &. & & $r$ & . & &. & + & + & . & . & &. & & $\dot{.}$ & 9 & 35 \\
\hline & Fagus sylvatica & E1 & . & & & . & & . & . & & . & . & 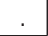 & + & . & &. & . & & . & . & & . & . & & . & & . & 1 & 4 \\
\hline & Galium laevigatum & E1 & . & + & & . & & & + & & . & . & + & + & . & & . & 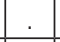 & & + & & & $\dot{0}$ & + & & . & & & 6 & 23 \\
\hline & Prenanthes purpurea & E1 & . & & & . & & & . & + & 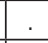 & + & &. & + & &. & + & &. &. & & . & + & &. & & + & 6 & 23 \\
\hline & Laburnum alpinum & E3a & . & & & . & & . & . & + & . & . & & . & . & &. & . & & . & . & & . & . & & . & & . & 1 & 4 \\
\hline & Laburnum alpinum & E2a &. & & & . & & & . & + & $\dot{\square}$ & . & &. &. & & 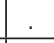 & . & &. & & & - & & & . & & & 1 & 4 \\
\hline & Laburnum alpinum & E1 &. & & & . & & &. & & $\dot{.}$ & . & + & . &. & & . & & &. &. & & . & & &. & & & 1 & 4 \\
\hline & Lilium martagon & E1 & . & + & & . & & & . & & . & . & & . & . & & $r$ & 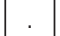 & & $r$ & . & & . & & & . & & . & 3 & 12 \\
\hline & Acer pseudoplatanus & E1 & & & & + & & & . & & & . & & . & . & & 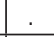 & . & & . & $\dot{.}$ & & 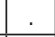 & & &. & & & 1 & 4 \\
\hline & Neottia nidus-avis & E1 & . & & & . & & & + & & 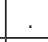 & & &. & . & &. & & &. & . & & . & & &. & &. & 1 & 4 \\
\hline & Dryopteris filix-mas & E1 & 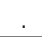 & & & . & & & . & & . & . & & . & . & & . & . & &. & . & & . & . & & + & & . & 1 & 4 \\
\hline QF & Querco-Fagetea & & & & & & & & & & & & & & & & & & & & & & & & & & & & & \\
\hline & Platanthera bifolia & E1 & & + & & $\mathrm{r}$ & & & . & + & + & & $\mathrm{r}$ & + & & + & + & + & + & + & & & $\dot{.}$ & & + & & & + & 13 & 50 \\
\hline & Carex digitata & E1 & & + & & & & & . & & & & & + & & & + & + & & + & + & & . & + & + & . & & & 8 & 31 \\
\hline
\end{tabular}




\begin{tabular}{|c|c|c|c|c|c|c|c|c|c|c|c|c|c|c|c|c|c|c|c|c|c|c|c|c|c|c|c|c|c|c|}
\hline & \multicolumn{2}{|c|}{$\begin{array}{l}\text { Number of relevé (Zaporedna } \\
\text { številka popisa) }\end{array}$} & 1 & 2 & 3 & 4 & 5 & 6 & 7 & 8 & 9 & 10 & 11 & 12 & 13 & 14 & 15 & 16 & 17 & 18 & 19 & 20 & 21 & 22 & 23 & 24 & 25 & 26 & Pr. & Fr. \\
\hline & Pyrus pyraster & E3a & & & 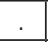 & & & 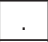 & $r$ & & & & & 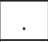 & & & & & & . & & & 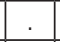 & & & . & & & 1 & 4 \\
\hline & Pyrus pyraster & $E 2 b$ & & & . & & + & + & & & 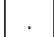 & & & . & & & . & & & . & & & . & & . & . & & & 2 & 8 \\
\hline & Pyrus pyraster & E2a & . & + & . & . & + &. & + & & . & . & . &. & & & . & & . &. & + & & . & 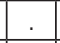 & . & . & + & . & 5 & 19 \\
\hline & Pyrus pyraster & E1 & & & . & & & + & & & & & &. & & & . & & - & . & & &. & & . & . & & & 1 & 4 \\
\hline & Carex montana & E1 & . & & - & & &. & & & & & + & + & & & + & + & . &. & & & $\cdot$ & & . & . & & . & 4 & 15 \\
\hline & Anemone nemorosa & E1 & . & 1 & . & . & . &. & . & & . & . & . &. & 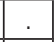 & . & . & 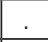 & . &. & & &. & . & . & + & & . & 2 & 8 \\
\hline & Cephalanthera Iongifolia & E1 & + & & . & & &. & + & &. & & 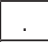 &. & & & . & & . &. & & &. & . & . & . & & . & 2 & 8 \\
\hline & Hedera helix & E3a & . & & . &. & . &. & . & &. &. & . &. & $\cdot$ & & . & . &. &. & & &. &. &. & . & & + & 1 & 4 \\
\hline & Hedera helix & E1 & . & $r$ & 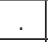 & + & 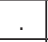 &. & . & 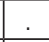 &. &. & . &. & 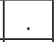 & . & . & 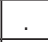 &. &. & & 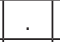 &. & . &. & . & &. & 2 & 8 \\
\hline & Malus sylvestris & E3b & . & & . & . & . &. & 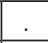 & &. &. &. &. & & & . & 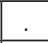 &. &. & & &. & . &. & . & & $r$ & 1 & 4 \\
\hline & Malus sylvestris & E2a & . & & . & . & + &. & . & + &. & $\cdot$ &. & . & & & . & . &. &. & & &. & . &. &. & & . & 2 & 8 \\
\hline & Rosa arvensis & E2a & + & . &. &. & . &. & . & 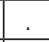 &. &. & . &. & . & . & . &. &. &. & 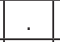 & . &. & . &. & + & & . & 2 & 8 \\
\hline & Corylus avellana & E2a & . & & $\cdot$ & $r$ & . & . & . & &. &. & . &. &. & . & . & . & $\cdot$ &. & . & . & $\cdot$ & . &. &. & & . & 1 & 4 \\
\hline & Corylus avellana & E1 &. & & . & + & &. & $\dot{.}$ & &. & ${ }_{0}$ & . &. & & & . & . & . &. & & &. & 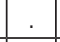 & . & . & & 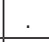 & 1 & 4 \\
\hline & Platanthera chlorantha & E1 & - & $r$ & . & . & . &. & . & &. & . & . &. & . & & . & 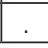 & . &. & & & . & - & . & . & & - & 1 & 4 \\
\hline EP & \multicolumn{30}{|l|}{ Erico-Pinetea } \\
\hline & $\begin{array}{l}\text { Molinia caerulea subsp. } \\
\text { arundinacea }\end{array}$ & E1 & 1 & 2 & 1 & 2 & 2 & 2 & 1 & 3 & 3 & 3 & 2 & 2 & 1 & 3 & 3 & 4 & 3 & 3 & 2 & 4 & 3 & 3 & 2 & 3 & 2 & 2 & 26 & 100 \\
\hline & Pinus nigra & E3b & . & & . & + & + &. & . & &. & . & $r$ &. & $\dot{.}$ & $r$ & $\dot{0}$ & &. &. & & & . & $\dot{0}$ &. & . & $r$ &. & 5 & 19 \\
\hline & Pinus nigra & E3a & . & &. & . & . &. & . & 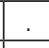 &. &. &. & $r$ & . & . & . &. &. &. & & 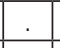 &. & . &. & . & . &. & 1 & 4 \\
\hline & Pinus nigra & $E 2 b$ & . & &. & . & 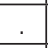 & . & . & &. &. & + &. & . & . & . & . &. &. & & &. & . &. & . & & . & 1 & 4 \\
\hline & Scleropodium purum & E0 & . & &. &. & + &. & . & 11 &. &. & + &. & . & + & . & &. &. & . & . &. & . &. & . & 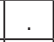 & . & 4 & 15 \\
\hline & Amelanchier ovalis & E2 & . & &. & . & . &. & . & & + & + & . & $r$ & . & & . & . &. &. & & . & . & . &. & . & + &. & 4 & 15 \\
\hline & Pinus sylvestris & E3b & . & & + & . & 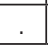 & . & . & &. & 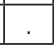 & 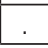 & $r$ & & & . & &. &. & & & . & - &. & . & $r$ & . & 3 & 12 \\
\hline VP & \multicolumn{30}{|l|}{ Vaccinio-Piceetea } \\
\hline & $\begin{array}{l}\text { Calamagrostis arundi- } \\
\text { nacea }\end{array}$ & E1 & 3 & 1 & 1 & 1 & + & + & + & + & 1 & 1 & + & 1 & 2 & . & + & 1 & 1 & 1 & 2 & 1 & 2 & 2 & 1 & 1 & 1 & + & 25 & 96 \\
\hline & Hypnum cupressiforme & E0 & 1 & + & . & & + & + & + & + & 1 & & + & 2 & 2 & 2 & 1 & 1 & + & 1 & + & + & + & + & + & + & + & + & 23 & 88 \\
\hline & $\begin{array}{l}\text { Avenella flexuosa (De- } \\
\text { schampsia flexuosa) }\end{array}$ & E1 & 1 & 1 & . & 1 & 1 & 1 & . & 1 & 1 & + & 1 & + & + & + & + & . & + & + & + & + & . & 1 & + & + & + & 1 & 22 & 85 \\
\hline & Luzula luzuloides & E1 &. & + &. & . &. & . & . & + & + & + & + & $r$ & + & . & + & + & + & + & + & + & + & + & + & + & + & + & 19 & 73 \\
\hline & Vaccinium myrtillus & E1 & 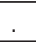 & & + & + & 2 & . & 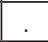 & 4 & 3 & 4 & 2 & 1 & 3 & 2 & 1 & 2 & & . & 2 & & + & & 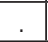 & . & & . & 14 & 54 \\
\hline & Hieracium murorum & E1 & + & + & . & + & &. &. & &. & 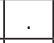 & . & + & - & + & + & + & + & + & & + & . & + & + & . & & + & 13 & 50 \\
\hline & Thuidium tamariscinum & E0 & . & + & . & $\cdot$ & & . & + & & + & & + & + & + & 1 & 1 & & . & 1 & & & . & & . & . & & + & 10 & 38 \\
\hline & $\begin{array}{l}\text { Polytrichum formosum } \\
\text { (Polytrichastrum formo- } \\
\text { sum) }\end{array}$ & E0 & + & + & . & + & + & . & & + & + & . & 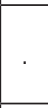 & + & . & . & . & & . & + & & . & . & . & . & . & & + & 9 & 35 \\
\hline & Atrichum undulatum & E0 & + & + & 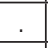 & + & & + & 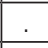 & . & + & 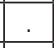 & + &. &. & . & . & & . & + & & & . & & + & . & & . & 8 & 31 \\
\hline & Pleurozium schreberi & E0 &. & & . & 2 & &. & & + &. & . & + & 1 & 1 & + & . & &. &. & . & & . & 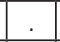 & . & . & & . & 6 & 23 \\
\hline & Solidago virgaurea & E1 & + & + & . & & &. & & & + & - & - &. & & & . & & . &. & + & & . & 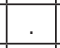 & + & . & & . & 5 & 19 \\
\hline & Leucobryum glaucum & E0 & + & + & . & 1 & . & . & . & &. &. &. &. & & + & . & . &. &. & & $\dot{i}$ & . & . &. & . & & . & 4 & 15 \\
\hline & Rhytidiadelphus triquetrus & E0 & $\dot{.}$ & &. &. & 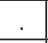 & . & $\dot{.}$ & &. & + & $\dot{\square}$ &. & & + & . & &. &. & & . & . & 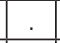 &. & . & & . & 2 & 8 \\
\hline & Aposeris foetida & E1 & - & + &. & . & &. & & &. & & - &. & & & . & & - &. & & & . & & . & . & & . & 1 & 4 \\
\hline & Picea abies & E3a & . & &. &. & & $r$ & & &. & $\pi$ &. &. & & & . & & . &. & & 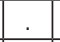 & . & $\cdot$ &. & . & & . & 1 & 4 \\
\hline & Dicranum scoparium & E0 & 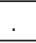 & &. & . & . &. & . & &. &. &. &. & $\dot{.}$ & - & . & . &. &. & & + &. & . &. &. & & . & 1 & 4 \\
\hline \multicolumn{31}{|c|}{\begin{tabular}{|l|l} 
& Rhamno-Prunetea, Sambuco-Salicion capreae
\end{tabular}} \\
\hline & Crataegus monogyna & E2 & + & + &. &. & 1. & + & + & &. &. & + &. & & $r$ & . & &. &. & & - &. &. &. &. & &. & 6 & 23 \\
\hline SSC & Sorbus aucuparia & E3b & . & $\dot{4}$ &. &. & . &. & . & $r$ &. &. & . &. & . & . & . & . &. &. & & 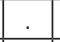 & . & . &. & . & . &. & 1 & 4 \\
\hline SSC & Sorbus aucuparia & E2a &. & &. & . & - & . & . & + &. &. & . &. & & - & . & 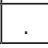 & . &. & & & . & . &. & . & & . & 1 & 4 \\
\hline \multicolumn{31}{|c|}{\begin{tabular}{l|l|}
$G$ & Trifolio-Geranietea \\
\end{tabular}} \\
\hline & Vincetoxicum hirundinaria & E1 & . & + &. & . & . & . & + & 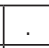 &. &. & . & . & $\dot{.}$ & . & . & . &. &. & &. & . & . &. & . & & . & 2 & 8 \\
\hline & Thesium bavarum & E1 &. & + & . & & &. & & &. & & &. & & & . & & & . & & & . & & . & . & & . & 1 & 4 \\
\hline & Anthericum ramosum & E1 & - & & . &. & &. & & &. & . & - &. & & & . & & . &. & & &. & & . & . & & + & 1 & 4 \\
\hline & Silene nutans & E1 & - & & . & . & . & . & . & &. &. & . & . & & & . & . & . &. & & &. &. &. & . & & + & 1 & 4 \\
\hline $\mathrm{FB}$ & Festuco-Brometea & & & & & & & & & & & & & & & & & & & & & & & & & & & & & \\
\hline & $\begin{array}{l}\text { Dianthus hyssopifolius (D. } \\
\text { monspessulanus) }\end{array}$ & E1 & + & + & . & $\cdot$ & . & . & + & & $\cdot$ & . & · & $\cdot$ & . & · & . & r & . & + & . & ' & + & . & . & . & + & + & 8 & 31 \\
\hline & Genista tinctoria & E1 & . & + & . & . & 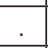 & . & + & &. &. & . & . & . & . & . & . & . & + & 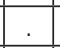 & . & . & + &. & . & & . & 4 & 15 \\
\hline & Peucedanum oreoselinum & E1 & + &. &. & . & - &. & . & &. & . & . &. & & & . & 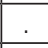 & &. & & & . & . &. & . & & . & 1 & 4 \\
\hline & Inula hirta & E1 & . & + & . &. & 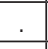 &. & & &. &. & 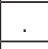 &. & & $\cdot$ & . & & &. & & &. & & $\cdot$ & . & & $\cdot$ & 1 & 4 \\
\hline & Hypochoeris maculata & E1 & . & & . & . & . & . & . & &. &. & . & . & . & - & $r$ & . & &. & & - & . & . &. & . & & . & 1 & 4 \\
\hline AT & Asplenietea trichomanis & & & & & & & & & & & & & & & & & & & & & & & & & & & & & \\
\hline & Polypodium vulgare & E1 & & & . & . & & . & & & . & . & & + & & & & $r$ & & + & & & . & & & . & & + & 4 & 15 \\
\hline
\end{tabular}




\begin{tabular}{|c|c|c|c|c|c|c|c|c|c|c|c|c|c|c|c|c|c|c|c|c|c|c|c|c|c|c|c|c|c|c|}
\hline & \multicolumn{2}{|c|}{$\begin{array}{l}\text { Number of relevé (Zaporedna } \\
\text { številka popisa) }\end{array}$} & \multirow[t]{2}{*}{1} & \multirow[t]{2}{*}{2} & \multirow[t]{2}{*}{3} & \multirow[t]{2}{*}{4} & \multirow[t]{2}{*}{5} & \multirow[t]{2}{*}{6} & \multirow[t]{2}{*}{7} & \multirow[t]{2}{*}{8} & \multirow[t]{2}{*}{9} & \multirow[t]{2}{*}{10} & \multirow[t]{2}{*}{11} & \multirow[t]{2}{*}{12} & \multirow[t]{2}{*}{13} & \multirow[t]{2}{*}{14} & \multirow[t]{2}{*}{15} & \multirow[t]{2}{*}{16} & \multirow[t]{2}{*}{17} & & \multirow[t]{2}{*}{19} & \multirow[t]{2}{*}{20} & \multirow[t]{2}{*}{21} & 22 & 23 & 24 & 25 & 26 & Pr. & Fr. \\
\hline & $\begin{array}{l}\text { Asplenium adiantum- } \\
\text { nigrum }\end{array}$ & E1 & & & & & & & & & & & & & & & & & & $r$ & & & & & & . & & + & 2 & 8 \\
\hline & Sedum maximum & E1 & 1 & &. & . & & . & . & &. & . & & . & . & & . & $r$ & & . & . & & . &. & & . & & & 1 & 4 \\
\hline & Asplenium trichomanes & E1 & . & & 4 & . & & 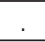 & . & &. & 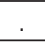 & & . & & & . & $r$ & & . & & &. & . & & . & & & 1 & 4 \\
\hline 0 & Other species (Druge vrs & & & & & & & & & & & & & & & & & & & & & & & & & & & & & \\
\hline & Juniperus communis & E2b & . & &. & + & + & 1 & 1 & & $r$ & . & & $r$ & + & & . & . & & . & . & & 1 & \begin{tabular}{|l|}
1 \\
\end{tabular} & & + & & & 10 & 38 \\
\hline & Juniperus communis & E2a & + & & 3 &. & + & + & 1 & &. & + & & + & + & $\mathrm{r}$ & $r$ & + & + & + & + & + & + & + & + & + & + & + & 22 & 85 \\
\hline & Aster sp. & E1 & . & & . & + & & 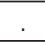 & . & &. & . & & . & . & & . & . & & . & 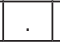 & &. & . & & . & & & 1 & 4 \\
\hline & Robinia pseudoacacia & E3 & . & &. & $\dot{.}$ & & . & . & &. &. & & . & . & & . & . & + & . & . & &. & . & & . & & & 1 & 4 \\
\hline & Robinia pseudoacacia & E2a & . & & . & 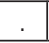 & & . & . & &. &. & & . & . & & . & . & + & . & . & &. & . & & . & & & 1 & 4 \\
\hline $\mathrm{ML}$ & Other mosses and lichen & (Dru & i ma & hovi & in liš & & & & & & & & & & & & & & & & & & & & & & & & & \\
\hline & Cladonia sp. & E0 &. & &. & + & & . & . & &. &. & + & + & + & + & + & . & & . & . & + &. & . & & . & & & 7 & 27 \\
\hline & Eurhynchium striatum & E0 & 1 & + &. &. & &. & . & & . &. & . & . & . & 1 & 1 & + & & 2 & . & &. & . & & . & 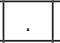 & . & 6 & 23 \\
\hline & Bryum capillare & E0 & . & &. &. & & + & . & & . & + & + & . & + & & . & . & & . & + & &. & . & & . & 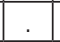 & . & 5 & 19 \\
\hline & Mnium sp. & E0 & . & &. &. & & & . & & . & . & & . & . & & + & + & & . & . & & + & . & & + & & & 4 & 15 \\
\hline & Isothecium alopecuroides & E0 & . & &. & + & & . & . & & . &. & & . & . & & + & . & & . & . & &. & . & & . & 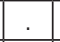 & + & 3 & 12 \\
\hline & Plagiothecium sp. & E0 & . & . &. &. & . &. & . & & . & + & & . & . & & . & . & & . & . & &. & . & & . & . & + & 2 & 8 \\
\hline & Tortella tortuosa & E0 & . & &. &. & & . & . & & . &. & & + & . & & + & . & & . & . & & . & . & & . & 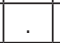 & & 2 & 8 \\
\hline & Fissidens taxifolius & E0 & . & &. & + & & . & . & & . &. & & . & . & & . & . & & . & . & &. & . & & . & . & & 1 & 4 \\
\hline & Ctenidium molluscum & E0 & . & . &. &. & + & . & . & & . &. & . & . & . & . & . & . & & . & . & . &. & . & & . & . & & 1 & 4 \\
\hline & Neckera crispa & E0 & . & . & . &. & 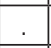 & . & . & & + &. & & . & . & . & . & . & & . & . & . & . & . & & . & . & . & 1 & 4 \\
\hline & Abietinella abietina & E0 &. & &. &. & & . & . & & . & + & & . & . & & . & . & & . & . & &. & . & & . & . & & 1 & 4 \\
\hline & Bryum sp. & E0 &. & &. &. & & . & . & &. &. & . & . & . & . & . & + & & . & . & &. & . & & . & . & & 1 & 4 \\
\hline & Rhodobryum roseum & E0 & . & &. & . & & r & . & & . &. & & . & & & . & . & & . & & & . & + & & & & & 1 & 4 \\
\hline
\end{tabular}

Legend - Legenda

Fly - Flysch / fliš

EU - Eutric brown soil / Evtrična rjava tla

CO Carpinion orientalis

EC Erythronio-Carpinion

CU Calluno-Ulicetea

MO Molinietalia caeruleae

SSC Sambuco-Salicion capreae 
Table 2: Seslerio autumnalis-Fagetum, Seslerio autumnalisQuercetum petraeae - Vipavska brda, Vrhe
Preglednica 2: Seslerio autumnalis-Fagetum, Seslerio autumnalis-Quercetum petraeae - Vipavska brda, Vrhe

\begin{tabular}{|c|c|c|c|c|c|c|c|c|c|c|c|c|c|c|c|c|c|c|c|}
\hline & Number of relevé (Zaporedna številka popisa) & & 1 & 2 & 3 & 4 & 5 & 6 & 7 & 8 & 9 & 10 & 11 & 12 & 13 & 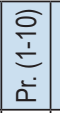 & 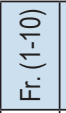 & 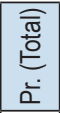 & 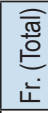 \\
\hline & Database number of relevé (Delovna številka popisa) & & 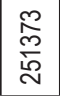 & 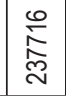 & 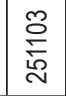 & 六 & 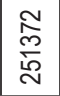 & 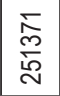 & 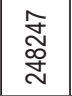 & $\begin{array}{l}\infty \\
\infty \\
\stackrel{m}{\leftarrow \sim} \\
\stackrel{\sim}{\sim}\end{array}$ & $\begin{array}{l}\underset{J}{J} \\
\text { o } \\
\text { d }\end{array}$ & 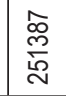 & 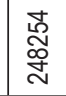 & 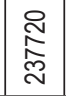 & 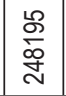 & & & & \\
\hline & Elevation in $\mathrm{m}$ (Nadmorska višina v $\mathrm{m}$ ) & & 330 & 580 & 400 & 450 & 440 & 370 & 440 & 380 & 440 & 385 & 320 & 440 & 360 & & & & \\
\hline & Aspect (Lega) & & $\mathrm{NE}$ & $\mathrm{N}$ & $\mathrm{NE}$ & $\mathrm{NE}$ & $\mathrm{NE}$ & $\mathrm{NE}$ & NNW & NW & $\mathrm{N}$ & $\mathrm{N}$ & $\mathrm{N}$ & $\mathrm{NE}$ & $\mathrm{NE}$ & & & & \\
\hline & Slope in degrees (Nagib v stopinjah) & & 35 & 20 & 25 & 30 & 25 & 25 & 25 & 20 & 20 & 20 & 15 & 10 & 20 & & & & \\
\hline & Parent material (Matična podlaga) & & Fly & Fly & Fly & Fly & Fly & Fly & Fly & Fly & Fly & Fly & Fly & Fly & Fly & & & & \\
\hline & Soil (Tla) & & $\mathrm{Eu}$ & $\mathrm{Eu}$ & $\mathrm{Eu}$ & $\mathrm{Eu}$ & $\mathrm{Eu}$ & $\mathrm{Eu}$ & $\mathrm{Eu}$ & $\mathrm{Eu}$ & $\mathrm{Eu}$ & $\mathrm{Eu}$ & $\mathrm{Eu}$ & $\mathrm{Eu}$ & Eu & & & & \\
\hline & Stoniness in \% (Kamnitost v \%) & & 10 & 10 & 5 & 5 & 10 & 10 & 5 & 10 & 20 & 5 & 0 & 0 & 20 & & & & \\
\hline & Cover in \% (Zastiranje $v \%$ ): & & & & & & & & & & & & & & & & & & \\
\hline & Upper tree layer (Zgornja drevesna plast) & E3b & 70 & 80 & 80 & 90 & 90 & 80 & 90 & 90 & 80 & 90 & 90 & 70 & 80 & & & & \\
\hline & Lower tree layer (Spodnja drevesna plast) & E3a & 20 & 10 & 10 & 5 & 5 & 20 & 10 & 5 & 10 & 5 & 10 & 30 & 0 & & & & \\
\hline & Shrub layer (Grmovna plast) & E2 & 20 & 10 & 10 & 5 & 5 & 20 & 5 & 10 & 30 & 10 & 20 & 10 & 20 & & & & \\
\hline & Herb layer (Zeliščna plast) & E1 & 70 & 70 & 70 & 60 & 50 & 60 & 30 & 60 & 30 & 70 & 80 & 100 & 60 & & & & \\
\hline & Moss layer (Mahovna plast) & E0 & 10 & 5 & 5 & 5 & 15 & 20 & 5 & 5 & 10 & 5 & 5 & 1 & 0 & & & & \\
\hline & Maximum diameter of trees (Največji prsni premer dreves) & $\mathrm{cm}$ & 40 & 40 & 35 & 40 & 40 & 35 & 35 & 50 & 35 & 35 & 30 & 30 & 40 & & & & \\
\hline & Maximum height of tress (Največja drevesna višina) & $\mathrm{m}$ & 18 & 24 & 18 & 20 & 22 & 19 & 22 & 26 & 24 & 22 & 20 & 14 & 26 & & & & \\
\hline & Number of species (Število vrst) & & 59 & 49 & 33 & 27 & 27 & 46 & 34 & 41 & 50 & 45 & 45 & 16 & 27 & & & & \\
\hline & Relevé area (Velikost popisne ploskve) & $\mathrm{m}^{2}$ & 400 & 400 & 400 & 400 & 400 & 400 & 400 & 200 & 400 & 200 & 400 & 400 & 200 & & & & \\
\hline & Locality (Nahajališče) & & 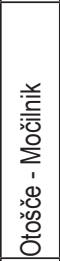 & 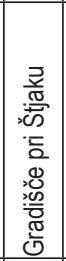 & 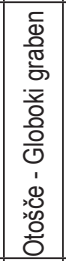 & 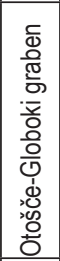 & 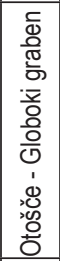 & 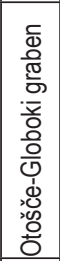 & 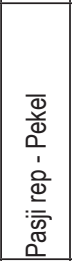 & 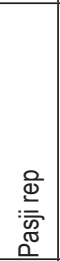 & 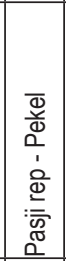 & 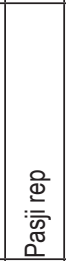 & 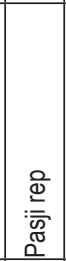 & 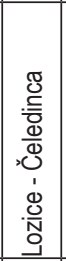 & 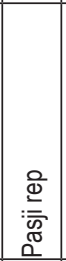 & & & & \\
\hline & Quadrant (Kvadrant) & & 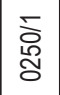 & 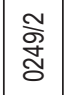 & 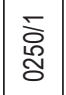 & 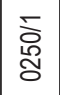 & 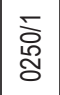 & 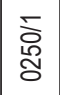 & 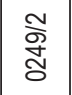 & 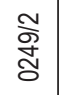 & 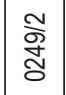 & 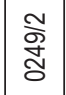 & 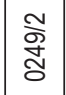 & 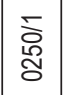 & \begin{tabular}{|c|}
\multirow{O}{*}{} \\
\multirow{\sigma}{\sigma}{} \\
\end{tabular} & & & & \\
\hline & Coordinate GK Y (D-48) & $\mathrm{m}$ & 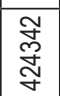 & 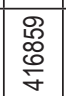 & 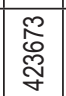 & 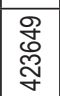 & 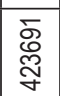 & \begin{tabular}{|l|}
\multirow{\sigma}{*}{} \\
$\underset{\sim}{\text { f }}$ \\
\end{tabular} & 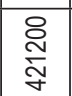 & 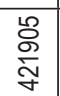 & 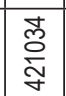 & 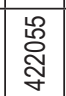 & 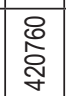 & 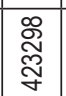 & 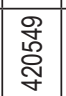 & & & & \\
\hline & Coordinate GK X (D-48) & $\mathrm{m}$ & 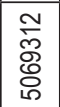 & 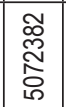 & 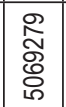 & $\begin{array}{l}\overline{8} \\
8 \\
o \\
\varnothing \\
\end{array}$ & \begin{tabular}{|l|} 
\\
$\&$ \\
$o$ \\
$o$ \\
0 \\
\end{tabular} & $\begin{array}{l}\overline{\widetilde{N}} \\
\mathbb{O} \\
\text { ¿ }\end{array}$ & 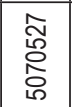 & 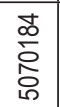 & $\begin{array}{l}\text { 㠻 } \\
\text { 总 } \\
\text { 员 }\end{array}$ & 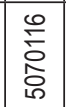 & 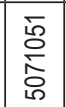 & \begin{tabular}{|l|} 
足 \\
o \\
o \\
in \\
\end{tabular} & \begin{tabular}{|l|}
8 \\
08 \\
$o 8$ \\
0 \\
\end{tabular} & & & & \\
\hline & \multicolumn{15}{|c|}{ Diagnostic species of the syntaxa (Diagnostične vrste sintaksonov) } & & & & \\
\hline FS & Fagus sylvatica & E3b & 4 & 5 & 5 & 5 & 5 & 5 & 4 & 4 & 4 & 4 & + & . & . & 10 & 100 & 11 & 85 \\
\hline FS & Fagus sylvatica & E3a & . & + & 1 & 1 & + & 1 & 1 & + & 1 & 1 & 1 & . & . & 9 & 90 & 10 & 77 \\
\hline FS & Fagus sylvatica & $E 2 b$ & 1 & 1 & 1 & + & + & 1 & 1 & + & 1 & + & + & $r$ & . & \begin{tabular}{|l|}
10 \\
\end{tabular} & 100 & 12 & 92 \\
\hline FS & Fagus sylvatica & E2a & $\cdot$ & & + & $\dot{.}$ & $\dot{.}$ & + & . & + & + &. & + & . & . & 4 & 40 & 5 & 38 \\
\hline FS & Fagus sylvatica & E1 & . & 1 & + & . & + & . & . & + & 1 & + & . & . & . & 6 & 60 & 6 & 46 \\
\hline $\mathrm{CO}$ & Sesleria autumnalis & E1 & 4 & 4 & 3 & 3 & + & + & 1 & 1 & 3 & 3 & 4 & 5 & . & \begin{tabular}{|l|}
10 \\
\end{tabular} & 100 & 12 & 92 \\
\hline $\mathrm{QP}$ & Lathyrus venetus & E1 & & & + & & & $r$ & + & + & 1 & + & 1 & . & . & 6 & 60 & 7 & 54 \\
\hline QR & Quercus petraea & E3b & 2 & 1 & + & 1 & $r$ & + & 1 & 1 & 1 & 2 & 5 & 4 & . & 10 & 100 & 12 & 92 \\
\hline$Q R$ & Quercus petraea & E3a & & & & & & + & . & . & . & . & . & 1 & . & 1 & 10 & 2 & 15 \\
\hline$Q R$ & Quercus petraea & E1 & & + & + & & & & + & . &. &. & 1 & 1 & . & 3 & 30 & 5 & 38 \\
\hline FS & Prunus avium & E3b & . & . & . & $r$ & . & . & 2 & . & $r$ & . & . & . & 4 & 3 & 30 & 4 & 31 \\
\hline FS & Prunus avium & E3a & & + & & & & & - & . & . & . & . & . & . & 1 & 10 & 1 & 8 \\
\hline FS & Prunus avium & E1 & & & + & + & + & & 1 & . & + & + & + & . &. & 6 & 60 & 7 & 54 \\
\hline \multirow[t]{3}{*}{$\mathrm{AF}$} & \multicolumn{19}{|l|}{ Aremonio-Fagion } \\
\hline & Lamium orvala & E1 & $r$ & & & $r$ & + & & 1 & 3 & + & . & . & . & 3 & 6 & 60 & 7 & 54 \\
\hline & Hacquetia epipactis & E1 & $r$ & & & & & $r$ & 1 & 1 & 1 & 1 & 1 & . & . & 6 & 60 & 7 & 54 \\
\hline EC & Primula vulgaris & E1 & + & + & . & . & . & . & + & + & . & 1 & + & . & + & 5 & 50 & 7 & 54 \\
\hline EC & Erythronium dens-canis & E1 & & & & . & & & 1 & . & 1 & + & $r$ & . & . & 3 & 30 & 4 & 31 \\
\hline & Anemone trifolia & E1 & 1 & & & & & & . & . & . & . & . & . & . & 1 & 10 & 1 & 8 \\
\hline & Anemone $x$ pittonii & E1 & + & . & . & . & . & & . & . & . & . & . & . & . & 1 & 10 & 1 & 8 \\
\hline & Euphorbia carniolica & E1 & + & & & & & & . & . & . & . & . & . & . & 1 & 10 & 1 & 8 \\
\hline & Calamintha grandiflora & E1 & & & & $r$ & & & . &. & . & . & . & . &. & 1 & 10 & 1 & 8 \\
\hline & Cradamine enneaphyllos & E1 & &. & . & . & + & $\dot{x}$ & . & . & . & . & . & . & . & 1 & 10 & 1 & 8 \\
\hline
\end{tabular}




\begin{tabular}{|c|c|c|c|c|c|c|c|c|c|c|c|c|c|c|c|c|c|c|c|}
\hline & Number of relevé (Zaporedna številka popisa) & & 1 & 2 & 3 & 4 & 5 & 6 & 7 & 8 & 9 & 10 & 11 & 12 & 13 & 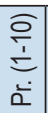 & 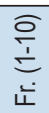 & 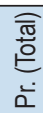 & 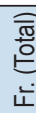 \\
\hline & Knautia drymeia & $\mathrm{E} 1$ & . & . & . & . & . & . & . & . & . & $r$ & . & . & . & 1 & 10 & 1 & 8 \\
\hline \multirow{40}{*}{ 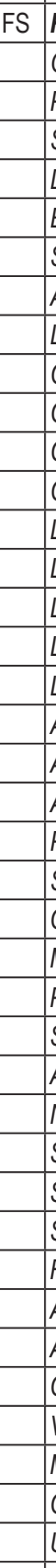 } & Fagetalia sylvaticae & & & & & & & & & & & & & & & & & & \\
\hline & Galium laevigatum & E1 & 2 & + & + & . & + & + & . & + & 1 & 1 & 1 & . &. & 8 & 80 & 9 & 69 \\
\hline & Prenanthes purpurea & E1 & . & 1 & 1 & 2 & 2 & 1 & 1 & + & 1 & 1 & . & . & . & 9 & 90 & 9 & 69 \\
\hline & Symphytum tuberosum & E1 & + & . & . & . & . & 1 & 1 & 1 & + & 1 & + & 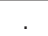 & + & 6 & 60 & 8 & 62 \\
\hline & Dryopteris filix-mas & E1 & . & 1 & + & + & . & . & + & + & + & . & + & . & 1 & 6 & 60 & 8 & 62 \\
\hline & Euphorbia dulcis & E1 & $r$ & . & + & . & . & $r$ & . & + & + & + & + & . & . & 6 & 60 & 7 & 54 \\
\hline & Salvia glutinosa & E1 & 1 & + & . & . & . & . & . & + & + & + & + & 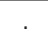 & + & 5 & 50 & 7 & 54 \\
\hline & Asarum europaeum subsp. caucasicum & E1 & . & . & . & . & . & . & + & 1 & + & 1 & + &. & + & 4 & 40 & 6 & 46 \\
\hline & Lilium martagon & $\mathrm{E} 1$ & + & . & . & . & . & + & + & . & + & + & . & . & + & 5 & 50 & 6 & 46 \\
\hline & Carpinus betulus & $E 3 b$ & . & . & $r$ & . & . & . & . & + & 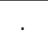 & 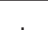 & . & . & . & 2 & 20 & 2 & 15 \\
\hline & Carpinus betulus & E3a & + & . & . & . & . & . & + & + & - & $r$ & + &. & . & 4 & 40 & 5 & 38 \\
\hline & Carpinus betulus & $E 2 b$ & $r$ & . & . & . & . & . & + & . & . & . & . & . & . & 2 & 20 & 2 & 15 \\
\hline & Carpinus betulus & E1 & . & . & . & . & . & . & . & + & 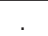 & . & . & . & + & 1 & 10 & 2 & 15 \\
\hline & Laburnum alpinum & E3a & + & . & . & . & $\mathrm{r}$ & $\mathrm{r}$ & . & & 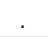 & 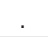 & . & 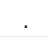 & 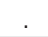 & 3 & 30 & 3 & 23 \\
\hline & Laburnum alpinum & $E 2 b$ & + & . & . & + & . & . & . & . & . & . & . & . & . & 2 & 20 & 2 & 15 \\
\hline & Laburnum alpinum & E2a & 1 & . & . & . & . & . & . & . & . & . & . & . & . & 1 & 10 & 1 & 8 \\
\hline & Laburnum alpinum & E1 & + & . & + & + & + & + & . & & & - & & 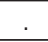 & 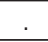 & 5 & 50 & 5 & 38 \\
\hline & Lathyrus vernus & $\mathrm{E} 1$ & + & . & . & . & . & $r$ & . & . & + & + & 1 & . & . & 4 & 40 & 5 & 38 \\
\hline & Acer pseudoplatanus & E3b & + & . & . & . & . & . &. & . & . & . & . & . & + & 1 & 10 & 2 & 15 \\
\hline & Acer pseudoplatanus & E2a & + & . & . & . & . & . & . & . & . & . & . & . & . & 1 & 10 & 1 & 8 \\
\hline & Acer pseudoplatanus & $\mathrm{E} 1$ & . & + & . & + & + & . & . & . & . & . & . & . & + & 3 & 30 & 4 & 31 \\
\hline & Pulmonaria officinalis & E1 & . & + & . & . & . & . & . & + & . & . & + & . & + & 2 & 20 & 4 & 31 \\
\hline & Senecio nemorensis & E1 & . & + & . & . & . & . & 1 & + & . & + & . & . & . & 4 & 40 & 4 & 31 \\
\hline & Campanula trachelium & E1 & . & + & . & . & . & . & + & . & . & + & . & . & . & 3 & 30 & 3 & 23 \\
\hline & Mycelis muralis & E1 & . & + & . & + & . & . & . & + & . & . & . & . & . & 3 & 30 & 3 & 23 \\
\hline & Polygonatum multiflorum & E1 & . & + & . & . & . & + & . & . & + & . & . & . & . & 3 & 30 & 3 & 23 \\
\hline & Sanicula europaea & E1 & . & . & . & . & . & . & . & + & + & + & . & . & . & 3 & 30 & 3 & 23 \\
\hline & Acer platanoides & E1 & . & . & . & + & + & . & . & . & . & . & . & . & . & 2 & 20 & 2 & 15 \\
\hline & Neottia nidus-avis & E1 & + & . & . & . & . & . & . & . & . & . & . & . & . & 1 & 10 & 1 & 8 \\
\hline & Sambucus nigra & $E 2 b$ & . & + & . & . & . & . & . & . & . & . & . & . & . & 1 & 10 & 1 & 8 \\
\hline & Sambucus nigra & E1 & . & . & . & . & . & . & . & . & . & . & . & . & + & 0 & 0 & 1 & 8 \\
\hline & Senecio ovatus (S. fuchsii) & E1 & . & + & . & . & . & . & . & . & . & . & . &. & . & 1 & 10 & 1 & 8 \\
\hline & Fraxinus excelsior & E3b & . & . & . & . & . & $r$ & . & . & . & . & . & . & . & 1 & 10 & 1 & 8 \\
\hline & Aruncus dioicus & E1 & . & . & . & . & . & + & . & . & . & . & . & . & . & 1 & 10 & 1 & 8 \\
\hline & Actaea spicata & E1 & . & . & . & . & . & . & + & 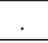 & . & . & . & . & + & 1 & 10 & 2 & 15 \\
\hline & Cardamine bulbifera & $\mathrm{E} 1$ &. & . & . & . & . & . & + & . & . & . & . & . & . & 1 & 10 & 1 & 8 \\
\hline & Viola reichenbachiana & $\mathrm{E} 1$ & . & . & . & . & . & . &. & + & . & . & . & . & . & 1 & 10 & 1 & 8 \\
\hline & Mercurialis perennis & E1 & . & . & . & . & . & . & . & & . & + & . & . & 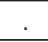 & 1 & 10 & 1 & 8 \\
\hline & Circaea lutetiana & E1 & . & . & . & . & . & . & . & . & . & . & . & . & 1 & 0 & 0 & 1 & 8 \\
\hline & Ulmus glabra & E1 & . & . & . & . & . & . &. & . & . & . & . & . & $r$ & 0 & 0 & 1 & 8 \\
\hline QP & \multicolumn{19}{|l|}{ Q Quercetalia pubescenti-petraeae } \\
\hline & Fraxinus ornus & E3a & 1 & + & . & + & . & + & + & + & . & + & 1 & 2 & + & 7 & 70 & 10 & 77 \\
\hline & Fraxinus ornus & $E 2 b$ & 1 & + & . & . & . & 1 & . & 5 & . & + & 2 & 2 & . & 4 & 40 & 6 & 46 \\
\hline & Fraxinus ornus & E2a & 2 & + & + & . & . & 1 & . & . & . & + & 1 & 1 & . & 5 & 50 & 7 & 54 \\
\hline & Fraxinus ornus & $\mathrm{E} 1$ & 1 & + & . & + & + & 1 & . & + & + & . & 1 & 1 & . & 7 & 70 & 9 & 69 \\
\hline & Tanacetum corymbosum & E1 & 1 & + & + & . & + & + & . & . & 1 & + & 1 & 1 & . & 7 & 70 & 9 & 69 \\
\hline & Campanula persicifolia & E1 & 1 & + & + & + & + & . & . & . & + & + & $r$ & . & . & 7 & 70 & 8 & 62 \\
\hline & Sorbus aria & E3b & . & . & . & . & . & + & . & . & . & . & . & . & . & 1 & 10 & 1 & 8 \\
\hline & Sorbus aria & E3a & . & + & . & + & . & + & . & . & + & . & + & + & . & 4 & 40 & 6 & 46 \\
\hline & Sorbus aria & $E 2 b$ & + & . & . & . & . & + & + & . & $r$ & + & 1 & + & . & 5 & 50 & 7 & 54 \\
\hline & Sorbus aria & E2a & + & + & . & . & . & + & . & . & + & + & . & + & . & 5 & 50 & 6 & 46 \\
\hline & Ostrya carpinifolia & E3b & 1 & + & . & . & . & . & + & . & + & . & . & . & + & 4 & 40 & 5 & 38 \\
\hline & Ostrya carpinifolia & E3a & 1 & . & . & . & . & . & . & 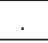 & . & + & $r$ &. &. & 2 & 20 & 3 & 23 \\
\hline & Ostrya carpinifolia & $E 2 b$ & + & . & . & . & . & . & . & . & . & . & . & . & . & 1 & 10 & 1 & 8 \\
\hline $\mathrm{CO}$ & Asparagus tenuifolius & $\mathrm{E} 1$ & . & + & . & . & . & . & . & + & + & & + & & & 3 & 30 & 4 & 31 \\
\hline & Melittis melissophyllum & $\mathrm{E} 1$ & 1 & . & . & . & . & . & . & + & & + & 1 & & & 3 & 30 & 4 & 31 \\
\hline & Sorbus torminalis & E3a & . & . & + & . & . & . & . & . & . & . & 1 & + & . & 1 & 10 & 3 & 23 \\
\hline & Sorbus torminalis & $E 2 b$ & . & . & . & . & . & . & . & & + & & + & & & 1 & 10 & 2 & 15 \\
\hline & Sorbus torminalis & E2a & . & . & + & . & . & + & . & & + & & 1 & & & 3 & 30 & 4 & 31 \\
\hline & Sorbus torminalis & E1 & . & . & + & . & . & . & . & & . & . & . & . & . & 1 & 10 & 1 & 8 \\
\hline
\end{tabular}




\begin{tabular}{|c|c|c|c|c|c|c|c|c|c|c|c|c|c|c|c|c|c|c|c|}
\hline & Number of relevé (Zaporedna številka popisa) & & 1 & 2 & 3 & 4 & 5 & 6 & 7 & 8 & 9 & 10 & 11 & 12 & 13 & 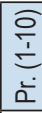 & 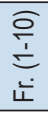 & 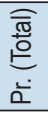 & 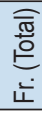 \\
\hline & Convallaria majalis & E1 & 1 & . & . & . & . & + & . & + & . & . & . & . & . & 3 & 30 & 3 & 23 \\
\hline & Lathyrus niger & E1 & 1 & . & . & . & . & . & . & . & . & . & . & + & . & 1 & 10 & 2 & 15 \\
\hline $\mathrm{CO}$ & Aristolochia lutea & E1 & & 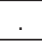 & . & 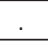 & . & - & + & + & . & . & & & . & 2 & 20 & 2 & 15 \\
\hline \multirow[t]{5}{*}{$\mathrm{FB}$} & Dianthus monspessulanus & E1 & + & . & . &. & . & . & . & . & . & . & . & . & . & 1 & 10 & 1 & 8 \\
\hline & Hypericum montanum & E1 & . & + & . & . & . & . & . & . & . & . & . & . & . & 1 & 10 & 1 & 8 \\
\hline & Arabis turrita & E1 &. & . & . & $r$ & . & . & . & . & . & . & . & . & . & 1 & 10 & 1 & 8 \\
\hline & Buglossoides purpurocaerulea & E1 & . & . & . & . & . & . & . & $r$ & . & . & . & . & . & 1 & 10 & 1 & 8 \\
\hline & Calamintha sylvatica & E1 & . & . & . & . & . & . & . & $\mathrm{r}$ & . & . & . & . & . & 1 & 10 & 1 & 8 \\
\hline \multirow[t]{4}{*}{ QR } & Quercetalia roboris & & & & & & & & & & & & & & & & & & \\
\hline & Hieracium racemosum & E1 & 1 & 1 & 1 & + & 1 & 1 &. & + & . & 1 & + & + & . & 8 & 80 & 10 & 77 \\
\hline & Serratula tinctoria & E1 & 1 & . & . & . & . & . & . & . & + & 1 & + & 2 & . & 3 & 30 & 5 & 38 \\
\hline & Hieracium sabaudum & E1 & + & . & . &. & . & + & . & . & 1 & . & . & . &. & 3 & 30 & 3 & 23 \\
\hline \multirow[t]{9}{*}{$A G$} & Alnus glutinosa & E3b &. &. &. &. & . & $r$ &. & $r$ & . & . & . & . &. & 2 & 20 & 2 & 15 \\
\hline & Castanea sativa & E3a & $r$ & . & . & . & . & . & . & . & . & . & . & . & . & 1 & 10 & 1 & 8 \\
\hline & Castanea sativa & $\mathrm{E} 2 \mathrm{~b}$ & . & + & . &. & . & . & . & . & . &. & . &. &. & 1 & 10 & 1 & 8 \\
\hline & Castanea sativa & E1 & + & + & . &. & . & . &. & . & . & . & . & . &. & 2 & 20 & 2 & 15 \\
\hline & Hieracium lachenalii & E1 & $r$ & . & . & . & . & . & & . & . & . & & & . & \begin{tabular}{|l|l}
1 & $r$
\end{tabular} & 10 & 1 & 8 \\
\hline & Lathyrus linifolius & E1 & + & 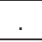 & . &. & . & . & . & . & . & . & . & . & . & 1 & 10 & 1 & 8 \\
\hline & Rubus hirtus & E2a & . & + & . & . & . & . & . & . & . & . & . & . & . & 1 & 10 & 1 & 8 \\
\hline & Melampyrum pratense subsp. vulgatum & E1 & . & . & . & . & . & 1 & . & . & . & . & & & . & 1 & 10 & 1 & 8 \\
\hline & Pteridium aquilinum & E1 & & & 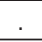 & & & &. & & + & & & & & 1 & 10 & 1 & 8 \\
\hline \multirow[t]{22}{*}{ QF } & Querco-Fagetea & & & & & & & & & & & & & & & & & & \\
\hline & Anemone nemorosa & E1 & + & 3 & . & 1 & + & + & 1 & 2 & 1 & 2 & + & & 1 & 9 & 90 & 11 & 85 \\
\hline & Carex digitata & E1 & & + & + & & . & & + & + & + & + & + & & + & 6 & 60 & 8 & 62 \\
\hline & Hedera helix & E3a &. & . & . &. & . & . &. & . & + & . & $r$ & . & . & 1 & 10 & 2 & 15 \\
\hline & Hedera helix & E1 & + & 1 & . & . & . & . & + & + & + & + & . & . & + & 6 & 60 & 7 & 54 \\
\hline & Aegopodium podagraria & E1 & & + & . & . & . & $r$ & . & 1 & & + & & . & + & 4 & 40 & 5 & 38 \\
\hline & Hepatica nobilis & E1 & $r$ & . & . &. &. &. &. & 1 & + & 1 & 1 &. & . & 4 & 40 & 5 & 38 \\
\hline & Acer campestre & E3b & . & . & . &. & . & . & . & $r$ & . & & . & . & $r$ & 1 & 10 & 2 & 15 \\
\hline & Acer campestre & E3a & $r$ &. & . & . & . & . & + & + & & + & & . & . & 4 & 40 & 4 & 31 \\
\hline & Acer campestre & E2a & + & . & . & . & . & . & . & . & . & + & . & . & . & 2 & 20 & 2 & 15 \\
\hline & Acer campestre & E1 & . & + & . & . & . & . & . & . & . & . & + & . & + & 1 & 10 & 3 & 23 \\
\hline & Corylus avellana & E3a & . &. & . & . & . & . & . & . & 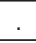 & & & & 2 & 0 & 0 & 1 & 8 \\
\hline & Corylus avellana & E2b &. &. & . &. &. & . & 1 & + & . & + & 1 & . & . & 3 & 30 & 4 & 31 \\
\hline & Corylus avellana & E2a & + & . & . & . & . & . & . & . & . & . & . & . & . & 1 & 10 & 1 & 8 \\
\hline & Cephalanthera Iongifolia & E1 &. & . & 1 &. & . & . & . & . & + & . &. & + &. & 2 & 20 & 3 & 23 \\
\hline & Crataegus laevigata & E2b & . & . & . & . & . & . & + & . & $r$ & . & . & . & . & 2 & 20 & 2 & 15 \\
\hline & Clematis vitalba & E1 & . & + & . & . & . & . & . & . & . & . & . & . & . & 1 & 10 & 1 & 8 \\
\hline & Moehringia trinervia & E1 & . & + & . &. & . & . & . & . & . & . & . & . & . & 1 & 10 & 1 & 8 \\
\hline & Pyrus pyraster & E2a & . &. &. &. & . & + &. &. &. &. & . & . &. & 1 & 10 & 1 & 8 \\
\hline & Dactylorhiza fuchsii & E1 & . & . & . & . & . & + & . & . & . & . & . & . & . & 1 & 10 & 1 & 8 \\
\hline & Rosa arvensis & E2a & . & . & . & . & . & . & & . & + &. & . & . & . & 1 & 10 & 1 & 8 \\
\hline & Platanthera chlorantha & E1 & . & . & . & . & . & . & . & . & . &. & $r$ & . &. & 0 & 0 & 1 & 8 \\
\hline \multirow[t]{15}{*}{ VP } & Vaccinio-Piceetea & & & & & & & & & & & & & & & & & & \\
\hline & Calamagrostis arundinacea & E1 & 1 & . & 1 & 1 & 2 & 2 & . & . & + & + & . & 1 & . & 7 & 70 & 8 & 62 \\
\hline & Aposeris foetida & E1 & . & . & 1 & . & . & + & 1 & 1 & 1 & 1 & + & . & . & 6 & 60 & 7 & 54 \\
\hline & Hieracium murorum & E1 & 1 & + & 1 & 1 & 1 & 1 & . & . & 1 & & & & . & 7 & 70 & 7 & 54 \\
\hline & Luzula luzuloides & E1 & + & 1 & 1 & 1 & 2 & 1 &. & . & . & 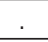 & + & &. & 6 & 60 & 7 & 54 \\
\hline & Avenella flexuosa (Deschampsia flexuosa) & E1 & 1 & + & + & + & 1 & 1 & . & . & . & . & . & . & . & 6 & 60 & 6 & 46 \\
\hline & Atrichum undulatum & E0 & + & + & + & + & . & & . & . & + & & & & . & 5 & 50 & 5 & 38 \\
\hline & Solidago virgaurea & E1 & + & + & + &. & + & . & . & . & . & . & 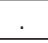 & . &. & 4 & 40 & 4 & 31 \\
\hline & Hypnum cupressiforme & E0 & 1 & . & + & . & . & 1 & . & . & . & + & . & . & . & 4 & 40 & 4 & 31 \\
\hline & Veronica urticifolia & E1 & . & . & . & + & + & + & . & 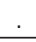 & . & $\dot{.}$ & & & . & 3 & 30 & 3 & 23 \\
\hline & Polytrichum formosum (Polytrichastrum formosum) & E0 & & 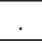 & + & + & 1 & . & . & . & 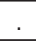 & . & & & & 3 & 30 & 3 & 23 \\
\hline & Gentiana asclepiadea & E1 & . & . & . & . & . & + & . & . & + & . & . & . & . & 2 & 20 & 2 & 15 \\
\hline & Abies alba & E3a & . & . & . & . & . & . & . & . & & $\dot{.}$ & & & $r$ & 0 & 0 & 1 & 8 \\
\hline & Abies alba & E2a & & + & . & . & . & . & . & . & & 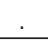 & & & & 1 & 10 & 1 & 8 \\
\hline & Vaccinium myrtillus & E1 & . & . & . & . & . & + & . & . & . & . & . & . & . & 1 & 10 & 1 & 8 \\
\hline & Thuidium tamariscinum & E0 & . & & . & & & + & . & . & $\cdot$ & & & & & 1 & 10 & 1 & 8 \\
\hline \multirow[t]{2}{*}{ EP } & Erico-Pinetea & & & & & & & & & & & & & & & & & & \\
\hline & Erica carnea & E1 & 1 & . & . & . & . & + &. & . & . & . & . & + & . & 2 & 20 & 3 & 23 \\
\hline
\end{tabular}




\begin{tabular}{|c|c|c|c|c|c|c|c|c|c|c|c|c|c|c|c|c|c|c|c|}
\hline & Number of relevé (Zaporedna številka popisa) & & 1 & 2 & 3 & 4 & 5 & 6 & 7 & 8 & 9 & 10 & 11 & 12 & 13 & 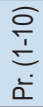 & 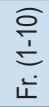 & 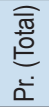 & 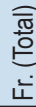 \\
\hline & Chamaecytisus hirsutus & E1 & + & . & . & . & . & . & . & . & . & . & . & . & . & 1 & 10 & 1 & 8 \\
\hline & Molinia caerulea subsp. arundinacea & E1 & . & . & . & . & . & . & . & . & . & . & . & + & . & 0 & 0 & 1 & 8 \\
\hline \multirow[t]{6}{*}{$\mathrm{RP}$} & Rhamno-Prunetea & & & & & & & & & & & & & & & & & & \\
\hline & Crataegus monogyna & E3a & + & . & 5 & . & . & . & . & . & . & . & . & . & . & 1 & 10 & 1 & 8 \\
\hline & Crataegus monogyna & E2a & . & + & . & . & . & . & + & . & + & + & . & + & . & 4 & 40 & 5 & 38 \\
\hline & Crataegus monogyna & $E 2 b$ & . & . & . & & . & . & . & + & + & + & + & & . & 3 & 30 & 4 & 31 \\
\hline & Ligustrum vulgare & E2a & & + & & & & & . & . & . & . & . & . & . & 1 & 10 & 1 & 8 \\
\hline & Rubus fruticosus agg. & E1 & . & . & . & 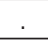 & . & . & . & . & . & . & . & . & + & 0 & 0 & 1 & 8 \\
\hline \multirow[t]{4}{*}{ TG } & Trifolio-Geranietea & & & & & & & & & & & & & & & & & & \\
\hline & Campanula rapunculoides & E1 & 2 & & + & & & + & + & + & 1 & 1 & 1 & . & . & 7 & 70 & 8 & 62 \\
\hline & Vincetoxicum hirundinaria & E1 & . & . & . & & . & . & . & . & + & + & 1 & . & . & 2 & 20 & 3 & 23 \\
\hline & Iris graminea & E1 & . & . & . & & & . & . & . & . & . & + & . & . & 0 & 0 & 1 & 8 \\
\hline \multirow[t]{3}{*}{ AT } & Asplenietea trichomanis & & & & & & & & & & & & & & & & & & \\
\hline & Polypodium vulgare & E1 & 1 & 1 & + & + & 1 & + & . & . & + & + & + & . & . & 8 & 80 & 9 & 69 \\
\hline & Asplenium trichomanes & E1 & + & . & . & . & . & . & . & . & + & . & + & . & . & 2 & 20 & 3 & 23 \\
\hline 0 & Other species (Druge vrste) & & & & & & & & & & & & & & & & & & \\
\hline \multirow[t]{8}{*}{ MA } & Veronica chamaedrys & E1 & . & + & . & . & . & . & . & . & . & . & . & . & . & 1 & 10 & 1 & 8 \\
\hline & Juglans regia & E3b & . & . & . & . & . & . & . & . & . & . & . & . & 1 & 0 & 0 & 1 & 8 \\
\hline & Juniperus communis & E2a & . & . & . & . & . & . & . & . & . & . & . & + & . & 0 & 0 & 1 & 8 \\
\hline & Juniperus communis & $E 2 b$ & . & . & . & . & . & . & . & . & . & . & . & + & . & 0 & 0 & 1 & 8 \\
\hline & Robinia pseudoacacia & E3a & $r$ & . & . & . & . & . & . & . & . & . & . & . & . & 1 & 10 & 1 & 8 \\
\hline & Robinia pseudoacacia & E3b & . & . & . & . & . & . & . & . & . & . & . & . & $r$ & 0 & 0 & 1 & 8 \\
\hline & Robinia pseudoacacia & $\mathrm{E} 2 \mathrm{~b}$ & $r$ & . & . & . & . & . & . & . & . & . & . & . & . & 1 & 10 & 1 & 8 \\
\hline & Solanum dulcamara & E1 & . & + & . & . & . & . & . & . & . & . & . & . & . & 1 & 10 & 1 & 8 \\
\hline GU & Geum urbanum & E1 & & + & . & - & & . & . & . & . & . & . & . & . & 1 & 10 & 1 & 8 \\
\hline \multirow[t]{16}{*}{$\mathrm{ML}$} & Other mosses (Drugi mahovi) & & & & & & & & & & & & & & & & & & \\
\hline & Isothecium alopecuroides & E0 & 1 & + & + & . & . & + & + & + & + & + & + & . & . & 8 & 80 & 9 & 69 \\
\hline & Schistidium apocarpum & E0 & + & + & . & . & + & . & + & + & + & . & . & . & . & 6 & 60 & 6 & 46 \\
\hline & Peltigera canina & E0 & + & . & + & . & . & . & . & . & . & . & . & . & . & 2 & 20 & 2 & 15 \\
\hline & Eurhynchium striatum & E0 & + & . & . & . & . & . & . & . & . & . & + & . & . & 1 & 10 & 2 & 15 \\
\hline & Plagiothecium denticulatum & E0 & . & . & . & + & + & . & . & . & . & . & . & . & . & 2 & 20 & 2 & 15 \\
\hline & Neckera crispa & E0 & . & . & - & & 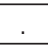 & + & . & . & + & . & . & . & . & 2 & 20 & 2 & 15 \\
\hline & Fissidens taxifolius & E0 & . & . & . & & . & + & . & . & . & . & + & . & . & 1 & 10 & 2 & 15 \\
\hline & Tortella tortuosa & E0 & + & . & . & & . & . & . & . & . & . & . & . & . & 1 & 10 & 1 & 8 \\
\hline & Marchantia polymorpha & E0 & & + & & & & & & . & . & . & . & . & . & 1 & 10 & 1 & 8 \\
\hline & Dicranum sp. & E0 & . & . & + & & . & . & . & . & . & . & . & . & . & 1 & 10 & 1 & 8 \\
\hline & Plagiochila porelloides & E0 & . & . & + & & 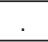 & . & . & . & . & . & . & . & . & 1 & 10 & 1 & 8 \\
\hline & Anomodon attenuatus & E0 & $\bar{c}$ & . & . & & + & 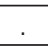 & . & . & . & . & . & . & . & 1 & 10 & 1 & 8 \\
\hline & Porella platyphylla & E0 & . & . & . & 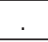 & . & + & . & . & . & . & . & . & . & 1 & 10 & 1 & 8 \\
\hline & Cladonia sp. & E0 & . & . & . & 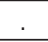 & . & + & . & . & . & . & . & . & . & 1 & 10 & 1 & 8 \\
\hline & Ctenidium molluscum & E0 & . & . & - & & & 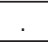 & - & . & + & . & . & . & . & 1 & 10 & 1 & 8 \\
\hline
\end{tabular}

Legend - Legenda

Fly - Flysch / fliš

EU - Eutric brown soil / Evtrična rjava tla

CO Carpinion orientalis

EC Erythronio-Carpinion

AG Alnetea glutinosae

FB Festuco-Brometea

MA Molinio-Arrhenetheretea

GU Galio-Urticetea 
Table 3: Synoptic table of the forest communities with dominant Quercus petraea in southwestern Slovenia and in northern Bosnia

\begin{tabular}{|c|c|c|c|c|c|c|c|}
\hline & Successive number (Zaporedna številka) & 1 & 2 & 3 & 4 & 5 & 6 \\
\hline & Number of relevés (Število popisov ) & 26 & 19 & 8 & 15 & 23 & 5 \\
\hline & Sign for syntaxa (Oznaka sintaksonov) & O & $\frac{O}{\Sigma}$ & $\begin{array}{ll}4 \\
\pi \\
0\end{array}$ & O & 邑 & $\bar{o}$ \\
\hline & \multicolumn{7}{|c|}{ Diagnostic species of the association Chamaecytiso-Quercetum } \\
\hline EP & Erica carnea & 100 & 5 & 38 & & 100 & 100 \\
\hline QP & Sorbus aria & 92 & 11 & 13 & 13 & & \\
\hline QP & Sesleria autumnalis & 85 & 5 & 93 & 93 & & 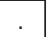 \\
\hline EP & Chamaecytisus hirsutus & 73 & & 50 & 33 & 30 & 40 \\
\hline QP & Loranthus europaeus & 62 & & . & & & . \\
\hline QR & Lathyrus linifolius & 69 & 5 & . & . & & . \\
\hline EC & Erythronium dens-canis & 50 & & . & & & 100 \\
\hline & \multicolumn{7}{|c|}{ Diagnostic species of the association Melampyro-Quercetum } \\
\hline QR & Melampyrum pratense subsp. vulgatum & 15 & 95 & 38 & 40 & & 40 \\
\hline QR & Chamaecytisus supinus & 19 & 95 & 25 & 13 & & . \\
\hline VP & Luzula luzuloides & 73 & 89 & 13 & & & . \\
\hline QR & Hieracium sabaudum & 96 & 89 & 25 & . & & . \\
\hline VP & Avenella flexuosa (Deschampsia flexuosa) & 85 & 89 & 100 & . & & . \\
\hline QR & Lembotropis nigricans & & 47 & 13 & . & & . \\
\hline FB & Orobanche ramosa subsp. nana & & 16 & & & & . \\
\hline & \multicolumn{7}{|c|}{$\begin{array}{l}\text { Diagnostic species of the association Seslerio autumnalis- } \\
\text { Quercetum petraeae }\end{array}$} \\
\hline $\mathrm{CO}$ & Knautia drymeia subsp. tergestina & & & 75 & 53 & & . \\
\hline $\mathrm{CO}$ & Helleborus odorus subsp. istriacus & . & & 38 & 27 & & . \\
\hline TG & Pulmonaria australis & & & 25 & 20 & & 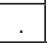 \\
\hline QR & Serratula tinctoria & 81 & 5 & 75 & 93 & 10 & 60 \\
\hline QR & Hieracium racemosum & 69 & 100 & 100 & 87 & &. \\
\hline FS & Salvia glutinosa & & & 75 & 33 & & \\
\hline QF & Carex digitata & 31 & & 25 & 27 & & 60 \\
\hline QF & Carex montana & 15 & 16 & 50 & 13 & & \\
\hline QF & Corylus avellana & 4 & 37 & 75 & 27 & & . \\
\hline FS & Carpinus betulus & . & 53 & 50 & 47 & & . \\
\hline $\mathrm{QP}$ & Campanula persicifolia & 8 & 11 & 38 & 13 & 30 & 60 \\
\hline FS & Lathyrus vernus & . & & 25 & 13 & & . \\
\hline TG & Digitalis grandiflora & . & 16 & 13 & 7 & & \\
\hline & \multicolumn{7}{|c|}{ Diagnostic species of the association Erico-Quercetum petraeae } \\
\hline$Q R$ & Potentilla alba &. & & 38 & 7 & 70 & 40 \\
\hline $\mathrm{AF}$ & Epimedium alpinum &. & & . & . & 70 & 80 \\
\hline TG & Trifolium alpestre & 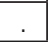 & & 50 & . & 50 & \\
\hline$Q R$ & Calluna vulgaris & 31 & 95 & 38 & 13 & 50 & 20 \\
\hline $\mathrm{QR}$ & Potentilla erecta & 42 & 68 & 50 & 53 & 50 & 20 \\
\hline FB & Genista tinctoria (incl. G. ovata) & 15 & 32 & 50 & 47 & 50 & 40 \\
\hline FS & Melica nutans & . & 5 & . & . & 50 & 80 \\
\hline FS & Euphorbia amygdaloides & . & & & 13 & 30 & \\
\hline$Q R$ & Veronica officinalis & . & 21 & 13 & . & 30 & \\
\hline QR & Danthonia decumbens & . & 11 & . & . & 30 & \\
\hline FS & Asarum europaeum & . & & & . & 30 & \\
\hline $\mathrm{AF}$ & Primula vulgaris & . & & 63 & 53 & 30 & \\
\hline QR & Agrostis tenuis & . & 21 & 38 & . & 30 & \\
\hline $\mathrm{QP}$ & Dianthus giganteus subsp. croaticus & . & & & . & 30 & \\
\hline FB & Danthonia alpina & . & & & . & 30 & \\
\hline VP & Luzula pilosa &. & . & . & . & 10 & \\
\hline TG & Centaurium erythraea & . & & & . & 10 & \\
\hline \multirow[t]{8}{*}{ QP } & \multicolumn{7}{|l|}{ Quercetalia pubescenti-petraeae } \\
\hline & Fraxinus ornus & 100 & 47 & 75 & 100 & 30 & 60 \\
\hline & Convallaria majalis & 92 & 53 & 38 & 20 & & . \\
\hline & Sorbus torminalis & 69 & 5 & 25 & 93 & 30 & 40 \\
\hline & Tanacetum corymbosum & 58 & 26 & 75 & 40 & 50 & 40 \\
\hline & Lathyrus niger & 23 & 63 & 63 & 100 & & . \\
\hline & Ostrya carpinifolia & 15 & . & 25 & 27 & 10 & . \\
\hline & Malus sylvestris & 8 & 5 & . & 27 & & . \\
\hline
\end{tabular}

Preglednica 3: Sintezna tabela gradnovih združb v jugozahodni Sloveniji in severni Bosni

\begin{tabular}{|c|c|c|c|c|c|c|c|}
\hline & Successive number (Zaporedna številka) & 1 & 2 & 3 & 4 & 5 & 6 \\
\hline & Sorbus austriaca s. lat. & 4 & & & & . & \\
\hline & Sorbus graeca & 4 & . & & & $\dot{.}$ & \\
\hline & Sorbus latifolia s. lat. & 4 &. & . & & . & . \\
\hline & Quercus cerris & & 63 & 50 & 33 &. & 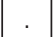 \\
\hline & Carex flacca & & 5 & 13 & 60 &. & \\
\hline & Buglossoides porpurocaerulea & . & 5 & 13 & 13 & . & . \\
\hline & Melittis melissophyllum & . & . & 63 & 53 & . & 40 \\
\hline & Hypericum montanum & & . & 50 & 7 & . & . \\
\hline & Cornus mas & . & . & 38 & 20 & . &. \\
\hline & Sorbus domestica & . & . & 13 & 40 & . &. \\
\hline & Ruscus aculeatus & & . & 13 & 33 & . & . \\
\hline & Quercus pubescens & . & . & 13 & 20 & . & . \\
\hline & Hierochloë australis & . & . & 13 & & . &. \\
\hline & Tamus communis & . & . & . & 47 & . &. \\
\hline $\mathrm{CO}$ & Lonicera etrusca & . & . & . & 40 & . & . \\
\hline $\mathrm{CO}$ & Asparagus tenuifolius & . &. & . & 27 & . &. \\
\hline & Carpinus orientalis & . &. &. & 20 & . &. \\
\hline $\mathrm{CO}$ & Cotinus coggygria & & . & . & 20 & 10 & . \\
\hline & Quercus x streimii & . & . & . & 13 & . &. \\
\hline & Orchis purpurea & & . & . & 13 & . & . \\
\hline & Rosa sempervirens & & . & . & 13 & . &. \\
\hline $\mathrm{CO}$ & Coronilla emeroides & . & . & . & 7 & . &. \\
\hline & Cephalanthera rubra & & . & . & & 30 & . \\
\hline & Acer tataricum & & . & . & & 30 & 20 \\
\hline & Prunus mahaleb & . & . & . & & 10 &. \\
\hline $\mathrm{CO}$ & Mercurialis ovata & & . & . & & 10 & . \\
\hline & Clematis recta & & . & . & & . & 40 \\
\hline & Pulmonaria mollisima & . &. & . & . & . & 60 \\
\hline QR & Quecetalia roboris, Calluno-Ulicetea & & & & & & \\
\hline & Quercus petraea & 100 & 100 & 88 & 100 & 100 & 100 \\
\hline & Genista pilosa & 65 & 42 & . & & 50 & 60 \\
\hline & Castanea sativa & 46 & 68 & 50 & 60 & 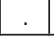 &. \\
\hline & Pteridium aquilinum & 42 & 58 & 13 & 20 & 30 & 100 \\
\hline & Hieracium lachenalii & 12 & . & . & & &. \\
\hline & Genista germanica & 4 & 74 & 25 & 20 & . &. \\
\hline & Phyteuma zahlbruckneri & 4 & 37 & . & & & . \\
\hline & Betonica officinalis $\mathrm{s}$. lat. & 4 & 26 & 75 & 80 & 50 & 80 \\
\hline & Betula pendula & 4 & 5 &. & . & . &. \\
\hline & Frangula alnus & . & 37 & . & . & 10 & 40 \\
\hline & Chamaespartium sagittale & . & 32 &. & . & . &. \\
\hline & Populus tremula & . & 16 &. & 13 & . &. \\
\hline $\mathrm{CU}$ & Nardus stricta & . & 16 & . & . & 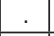 & . \\
\hline & Carex fritschii & . & & 38 & . & &. \\
\hline $\mathrm{CU}$ & Festuca filiformis & . & . & 25 & . & . & 40 \\
\hline & Rubus hirtus & & & . & . & 50 & 80 \\
\hline AF & Aremonio-Fagion, Erythronio-Carpinion & & & & & & \\
\hline EC & Lonicera caprifolium & . & . & 13 & 33 & . & . \\
\hline & Cyclamen purpurascens & . & & . & 13 & & \\
\hline & Daphne blagayana & . & & . & . & 30 & 20 \\
\hline & Aremonia agrimonoides & . & . &. & . & 30 & \\
\hline FS & Fagetalia sylvaticae & & & & & & \\
\hline & Fagus sylvatica & 35 & 53 & . & . & & . \\
\hline & $\begin{array}{l}\text { Galium laevigatum (incl. G. sylvaticum, G. } \\
\text { schultesii) }\end{array}$ & 23 & 11 & 38 & 40 & & 40 \\
\hline & Prenanthes purpurea & 23 & 32 & 13 & . & & $\dot{\square}$ \\
\hline & Lilium martagon & 12 & & 13 & 13 & & \\
\hline & Acer pseudoplatanus & 4 & 5 & & 13 & & \\
\hline & Laburnum alpinum & 4 & 5 & . & . & . & . \\
\hline & Neottia nidus-avis & 4 & & 13 & . & . & \\
\hline & Dryopteris filix-mas & 4 & & 13 & & & \\
\hline
\end{tabular}




\begin{tabular}{|c|c|c|c|c|c|c|c|}
\hline & Successive number (Zaporedna številka) & 1 & 2 & 3 & 4 & 5 & 6 \\
\hline & Viola reichenbachiana & . & 37 & 50 & 60 & & 60 \\
\hline & Polygonatum multiflorum & . & 16 & & 27 & . & . \\
\hline & Prunus avium & . & 5 & & 47 & 10 & . \\
\hline & Senecio fuchsii (incl. S. nemorensis) & . & 5 & 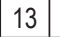 & & . & . \\
\hline & Euphorbia dulcis & . & & 38 & 60 &. & - \\
\hline & Symphytum tuberosum & . & . & 38 & 53 & . & 100 \\
\hline & Campanula trachelium & & . & 38 & 20 & . & \\
\hline & Heracleum sphondylium & . &. & 13 & 13 & . & . \\
\hline & Aruncus dioicus & . & . & 13 & & . & . \\
\hline & Brachypodium sylvaticum & & . & & 20 & & \\
\hline & Epipactis helleborine & . & & & 13 & & \\
\hline \multirow[t]{18}{*}{ QF } & \multicolumn{7}{|l|}{ Querco-Fagetea } \\
\hline & Platanthera bifolia & 50 & 16 & & 13 & & \\
\hline & Pyrus pyraster & 19 & 5 & 13 & 27 & . & 60 \\
\hline & Anemone nemorosa & 8 & 42 & 38 & 33 & . & 80 \\
\hline & Cephalanthera Iongifolia & 8 & & & 13 & . & \\
\hline & Hedera helix & 8 & 5 & 88 & 40 & . & . \\
\hline & Rosa arvensis & 8 & 21 & . & 33 & . & . \\
\hline & Platanthera chlorantha & 4 & & $\dot{.}$ & & (5 & . \\
\hline & Festuca heterophylla & & 63 & . & 67 & . & . \\
\hline & Cruciata glabra & & 16 & 50 & & 50 & 100 \\
\hline & Clematis vitalba & & 5 & 13 & 13 & . & . \\
\hline & Acer campestre & & . & 25 & 20 & . & . \\
\hline & Crataegus laevigata & & . & 13 & 47 & . & . \\
\hline & Ulmus minor & & . & . & 20 & . & . \\
\hline & Listera ovata & &. & . & 20 & . & . \\
\hline & Dactylorhiza fuchsii & . &. & . & 13 & . & . \\
\hline & Spiraea chamaedryfolia (S. ulmifolia) & . &. & . & & 30 & . \\
\hline & Viola riviniana & & $\cdot$ & . & & 30 & . \\
\hline EP & \multicolumn{7}{|l|}{ Erico-Pinetea s. lat. } \\
\hline & Molinia caerulea subsp. arundinacea & 100 & 26 & 38 & 33 & . & . \\
\hline & Scleropodium purum & 15 & 5 & . & & 30 & . \\
\hline & Amelanchier ovalis & 12 & & . & . & & . \\
\hline & Pinus sylvestris & 12 & 5 & . & . & . & . \\
\hline & Pinus nigra & 4 & . & 38 & . & . & 40 \\
\hline & Peucedanum austriacum & . & 16 & . & . & &. \\
\hline & Polygala chamaebuxus &. & 5 & . & & . &. \\
\hline & Calamagrostis varia & . &. & . & 13 & 10 & . \\
\hline & Scabiosa cinerea (S. leucophylla) &. & & . & . & 50 & 20 \\
\hline & Potentilla malyana &. &. & . & . & 50 &. \\
\hline & Festuca amethystina &. &. & . & . & 50 &. \\
\hline & Cytisus austriacus subsp. heuffelii &. & . & . & . & 30 &. \\
\hline & Cerastium moesiacum & . & & . & & 30 & . \\
\hline & Genista januensis &. &. & . & . & 30 &. \\
\hline & Cardamine plumieri & . & . & . & . & 30 & . \\
\hline & Viola beckiana & . & & . & . & 10 & 20 \\
\hline & Euphorbia montenegrina & $\cdot$ & . & . & . & . & 60 \\
\hline & Alyssum murale & . & . & . & . & & 20 \\
\hline & Rhamnus saxatilis & . & & . & $\dot{.}$ & & 20 \\
\hline & Sesleria latifolia & . & & . & . & & 20 \\
\hline \multirow[t]{13}{*}{ VP } & \multicolumn{7}{|l|}{ Vaccinio-Piceetea } \\
\hline & Calamagrostis arundinacea & 96 & 58 & 50 & 27 & & . \\
\hline & Hypnum cupressiforme & 88 & 95 & . & 20 & & . \\
\hline & Vaccinium myrtillus & 54 & 42 & . &. & 50 & 20 \\
\hline & Hieracium murorum & 50 & 79 & . & 27 & & . \\
\hline & Thuidium tamariscinum & 38 & 21 & . & 20 & & . \\
\hline & $\begin{array}{l}\text { Polytrichum formosum (Polytrichastrum } \\
\text { formosum) }\end{array}$ & 35 & 84 & 50 & . & $\cdot$ & \\
\hline & Atrichum undulatum & 31 & 47 & & . & $\cdot$ & . \\
\hline & Pleurozium schreberi & 23 & 47 & & & & \\
\hline & Solidago virgaurea & 19 & 74 & 50 & 47 & . & \\
\hline & Leucobryum glaucum & 15 & 79 & 38 & & $\cdot$ & \\
\hline & Rhytidiadelphus triquetrus & 8 & & & & & \\
\hline & Aposeris foetida & 4 & 5 & & & & \\
\hline
\end{tabular}

\begin{tabular}{|c|c|c|c|c|c|c|c|}
\hline & Successive number (Zaporedna številka) & 1 & 2 & 3 & 4 & 5 & 6 \\
\hline & Picea abies & 4 & 37 & . & . & . & . \\
\hline & Dicranum scoparium & 4 & 11 & 25 & . & & \\
\hline & Maianthemum bifolium & . & 11 & & . & & \\
\hline & Gentiana asclepiadea & . & 11 & 13 & . & . & . \\
\hline & Veronica urticifolia & . & 5 & & . & & \\
\hline & Luzula multiflora agg. &. & & 38 & . & & \\
\hline & Laserpitium krapfii (L. marginatum) & . & . & & . & 50 & 80 \\
\hline & Rosa pendulina &. & & & . & 30 & \\
\hline & Melampyrum sylvaticum & . & & & . & 30 & \\
\hline 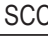 & Sambuco-Salicion capreae & & & & & & \\
\hline & Sorbus aucuparia & 4 & 11 & & . & & \\
\hline & Fragaria vesca & & 42 & 25 & 67 & & 40 \\
\hline $\mathrm{RP}$ & Rhamno-Prunetea & & & & & & \\
\hline & Crataegus monogyna & 15 & 16 & 75 & 80 & . & \\
\hline & Viburnum opulus & . & 11 & & & & \\
\hline & Rubus bifrons &. & 5 & 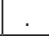 & 27 & . & . \\
\hline & Cornus sanguinea &. & . & 38 & 60 & . & . \\
\hline & Prunus spinosa &. & . & 38 & 40 & . & , \\
\hline & Euonymus europaea &. & . & 25 & 13 & . & . \\
\hline & Viburnum lantana & . & . & 13 & . & . & . \\
\hline & Rubus tomentosus & . & . & & 13 & . & \\
\hline & Rhamnus catharticus &. & . & . & 7 & 10 & . \\
\hline & Rosa spinossisima & . & . & . & . & 10 & . \\
\hline TG & Trifolio-Geranietea & & & & & & \\
\hline & Vincetoxicum hirundinaria & 8 & . & 38 & 73 & . & . \\
\hline & Anthericum ramosum & 4 & 11 & & 53 & . & . \\
\hline & Silene nutans & 4 & . & 50 & . & . & . \\
\hline & Thesium bavarum & 4 & . & . & . & . & . \\
\hline & Silene italica & . & 42 & . & . & . & . \\
\hline & Hypericum perforatum & . & 16 & & . & . & . \\
\hline & Calamintha brauneana & . & 11 & 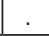 & . & . & . \\
\hline & Clinopodium vulgare &. & 5 & 38 & . & . & 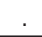 \\
\hline & Ligustrum vulgare & . & 5 & 13 & 73 & . & \\
\hline & Melampyrum nemorosum & . & 5 & & 13 & . & . \\
\hline & Trifolium medium & . & 5 & & & . & 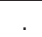 \\
\hline & Trifolium rubens & . & . & 13 & 47 & . & \\
\hline & Viola hirta &. & . & 25 & 20 & . & . \\
\hline & Euphorbia angulata & . & . & 13 & 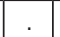 & . & . \\
\hline & Lilium bulbiferum & . & . & 13 & 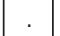 & . & . \\
\hline & Limodorum abortivum &. & . & 13 & . & . & . \\
\hline & Peucedanum cervaria &. & . &. & 20 & . & . \\
\hline & Vicia sylvatica & . & . & & 13 & . & . \\
\hline & Libanotis sibirica subsp. montana & . & . & . & 13 & . & . \\
\hline & Thalictrum minus & . & . & . & 7 & . & . \\
\hline $\mathrm{FB}$ & Festuco-Brometea & & & & & & \\
\hline & Dianthus hyssopifolius (D. monspessulanus) & 31 & 26 & 38 & . & . & . \\
\hline & Hypochoeris maculata & 4 & . & 38 & 20 & . & . \\
\hline & Inula hirta & 4 & & & & & \\
\hline & Peucedanum oreoselinum & 4 & . & 25 & 20 & 30 & 80 \\
\hline & Thymus serpyllium agg. (incl. Thymus sp.) & . & 26 & . & . & 70 & 80 \\
\hline & Prunella grandiflora & . & 11 & & . & & \\
\hline & Trifolium montanum & . & 11 & . & . & . & . \\
\hline & Dorycnium gremanicum & . & 5 & & . & 50 & \\
\hline & Genista sericea & . & 5 & & . & & \\
\hline & Pimpinella saxifraga &. & 5 & 13 & . & . & . \\
\hline & Cirsium acaule & . & 5 & & . & . & \\
\hline & Dianthus carthusianorum & . & 5 & & . & & \\
\hline & Globularia elongata &. & 5 & . & . & . & . \\
\hline & Koeleria sp. (Iobata, macrantha) & . & 5 & . & . & 30 & \\
\hline & Brachypodium pinnatum agg. & & & 63 & 40 & 30 & \\
\hline & Carex humilis &. & . & 13 & 40 & . & . \\
\hline & Filipendula vulgaris & . & & 50 & 27 & & \\
\hline & Teucrium chamaedrys & . & & 13 & 7 & & \\
\hline
\end{tabular}




\begin{tabular}{|c|c|c|c|c|c|c|c|}
\hline & Successive number (Zaporedna številka) & 1 & 2 & 3 & 4 & 5 & 6 \\
\hline & Allium carinatum subsp. pulchellum & & . & 13 & . & 50 & \\
\hline & Bromus erectus agg. & . & . & 13 & & 10 & 20 \\
\hline & Cirsium pannonicum & . & . & 13 & & & \\
\hline & Asphodelus albus & . & . & & 13 & & . \\
\hline & Euphorbia cyparisias & . & . & & 13 &. & \\
\hline & Centaurea triumfetti & & . & . & . & 30 & 20 \\
\hline & Teucrium montanum & & . & & . & 30 & . \\
\hline & Galium verum & & . & & . & 10 & \\
\hline & Galiium lucidum & & . & . & . & . & 80 \\
\hline MA & \multicolumn{7}{|c|}{ Molinio-Arrhenetheretea, Molinietalia caeruleae } \\
\hline $\mathrm{MO}$ & Laserpitium prutenicum & 8 & . & . & . &. & . \\
\hline & Veronica chamaedrys & & 37 & 50 & 33 & 30 & \\
\hline & Anthoxanthum odoratum & & 21 &. & . &. & . \\
\hline & Achillea millefolium & & 16 & . & . &. & . \\
\hline & Leontodon hispidus & & 11 & . & . & . & . \\
\hline & Ajuga reptans & & 5 & . & 27 &. & . \\
\hline & Centaurea jacea & & 5 & . & & . & . \\
\hline & Vicia sepium & & 5 & . & . & . & . \\
\hline & Leucanthemum vulgare & & . & . & 53 & . & . \\
\hline & Dactylis glomerata & & . & 25 & 33 & . & . \\
\hline & Vicia cracca & & . & 13 & 13 & 30 & 80 \\
\hline MO & Succisa pratensis & & . & 25 & & . & . \\
\hline & Galium mollugo & & . & . & 20 & . & . \\
\hline MO & Inula salicina & & . & . & 7 & . & . \\
\hline & Poa pratensis & & . & . & . & 70 & . \\
\hline & Centaurea stenolepis & & . & . & & 30 & . \\
\hline & Lathyrus pratensis & & . & . & & 30 & 80 \\
\hline & Carex caryophyllea & & . & . & . & 30 & . \\
\hline & $\begin{array}{l}\text { Centaurea nigrescens (inc. subsp. } \\
\text { smolinensis) }\end{array}$ & & & - & & 10 & 20 \\
\hline
\end{tabular}

\begin{tabular}{|c|c|c|c|c|c|c|c|}
\hline & Successive number (Zaporedna številka) & 1 & 2 & 3 & 4 & 5 & 6 \\
\hline & Lotus corniculatus & & . & & & . & 20 \\
\hline AT & \multicolumn{7}{|l|}{ Asplenietea trichomanis } \\
\hline & Polypodium vulgare & 15 & 5 & 25 & 20 & . & \\
\hline & Asplenium adiantum-nigrum & 8 & . & & & 10 & \\
\hline & Sedum maximum & 4 & . & 13 & & . & \\
\hline & Asplenium trichomanes & 4 & . & . & & . & . \\
\hline 0 & \multicolumn{7}{|l|}{ Other species (Druge vrste) } \\
\hline & Juniperus communis & 85 & 89 & 75 & 60 & . & \\
\hline & Robinia pseudoacacia & 4 & . & 25 & 27 & . & \\
\hline & Thlaspi kovatsii (T. avalanum) & & . &. & & 10 & . \\
\hline & Carduus carduelis & & 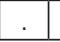 & . & & . & 60 \\
\hline ML & \multicolumn{7}{|c|}{ Other mosses and lichens (Drugi mahovi in lišaji) } \\
\hline & Cladonia sp. & 27 & 16 & . & 13 &. & . \\
\hline & Eurhynchium striatum & 23 & . & . & 33 &. & \\
\hline & Bryum capillare & 19 & . & . & . &. & . \\
\hline & Mnium sp. & 15 & . & . & . &. & . \\
\hline & Isothecium alopecuroides & 12 & 5 & . & &. & . \\
\hline & Plagiothecium sp. & 8 & . & . & . &. & . \\
\hline & Tortella tortuosa & 8 & . & . & . &. & . \\
\hline & Rhodobryum roseum & 4 & . & . & &. & . \\
\hline & Bryum sp. & 4 & . & . & &. & . \\
\hline & Ctenidium molluscum & 4 & . & . & 13 &. & . \\
\hline & Fissidens taxifolius & 4 & . & . & 13 &. & . \\
\hline & Neckera crispa & 4 & . & . & . &. & . \\
\hline & Thuidium abietinum & 4 & . & . & . &. & . \\
\hline & Thudium delicatulum & & 11 & . & &. & . \\
\hline & Cladonia rangiferina & & 11 & . & & . & . \\
\hline & Homlothecium lutescens & & 5 & . & &. & . \\
\hline
\end{tabular}

1 Chamaecytiso hirsuti-Quercetum petraeae ass. nov., this article

2 Melampyro vulgati-Quercetum petraeae Puncer \& Zupančič 1979 var. geogr. Fraxinus ornus (Puncer \& Zupančič) Zupančič 1994 (Puncer in Zupančič, 1979)

3 Seslerio autumnalis-Quercetum petraeae Poldini (1964) 1982 avenelletosum flexuosae Poldini 1982 (Poldini, 1982)

4 Seslerio autumnalis-Quercetum petraeae Poldini (1964) 1982 (Zupančič, 1999)

5 Erico-Quercetum petraeae Krause et Ludwig ex Horvat 1959 (Ritter-Studnička, 1970)

6 Erico-Quercetum petraeae Krause et Ludwig ex Horvat 1959 (Krause and Ludwig, 1957)

CO Carpinion orientalis, EC Erythronio-Carpinion, CU Calluno-Ulicetea, MO Molinietalia caeruleae

Table 4: Groups of diagnostic species in the stands of the associations Seslerio autumnalis-Fagetum, Chamaecytiso-Quercetum petraeae and Melampyro vulgati-Quercetum petraeae (relative frequencies)

\begin{tabular}{|l|c|c|c|}
\hline Successive number (Zaporedna številka) & 1 & 2 & 3 \\
\hline Sign for syntaxa (Oznaka sintaksonov) & SF & CQ & MQ \\
\hline Number of relevés (Število popisov) & 10 & 26 & 19 \\
\hline Quercetalia pubescenti-petraeae & 18 & 21 & 8,6 \\
\hline Quercetalia roboris, Calluno-Ulicetea & 8 & 27 & 36 \\
\hline Fagetalia sylvaticae, Aremonio-Fagion & 34 & 3,7 & $\mathbf{6 , 6}$ \\
\hline Querco-Fagetea & 12 & 7 & 6,5 \\
\hline Erico-Pinetea & 0,7 & 11 & 1,8 \\
\hline Vaccinio-Piceetea & 14 & 19 & 23 \\
\hline
\end{tabular}

Preglednica 4: Skupine diagnostičnih vrst v sestojih asociacij Seslerio autumnalis-Fagetum, Chamaecytiso-Quercetum petraeae in Melampyro vulgati-Quercetum petraeae (relativne frekvence)

\begin{tabular}{|l|c|c|c|}
\hline Successive number (Zaporedna številka) & 1 & 2 & 3 \\
\hline Rhamno-Prunetea, Sambuco-Salicion capreae & 1,5 & 0,7 & 2,5 \\
\hline Trifolio-Geranietea & 2,2 & 0,7 & 3,4 \\
\hline Festuco-Brometea & 0 & 2 & 4,5 \\
\hline Asplenietea trichomanis & 2,4 & 1,1 & 0,1 \\
\hline Molinio-Arrhenetheretea & 0 & 0 & 2,9 \\
\hline Other species (Druge vrste) & 1 & 3 & 2,6 \\
\hline Other mosses (Drugi mahovi) & 7,3 & 4,6 & 1,4 \\
\hline Skupaj (Total) & 100 & 100 & 100 \\
\hline
\end{tabular}

